DRAFT VERSION JunE 7, 2021

Preprint typeset using $\mathrm{L}^{\mathrm{A}} \mathrm{T}_{\mathrm{E}} \mathrm{X}$ style emulateapj v. 01/23/15

\title{
STAR CLUSTER FORMATION FROM TURBULENT CLUMPS.
}

\section{THE FAST FORMATION LIMIT}

\author{
Juan P. FArias ${ }^{1, \star}$, Jonathan C. TAN ${ }^{1}$ And Sourav ChatterjeE ${ }^{2}$ \\ ${ }^{1}$ Astronomy Department, University of Florida, Gainesville, FL 32611, USA \\ ${ }^{2}$ Center for Interdisciplinary Exploration \& Research in Astrophysics (CIERA) \\ Physics \& Astronomy, Northwestern University, Evanston, IL 60202, USA \\ Draft version June 7, 2021
}

\begin{abstract}
We investigate the formation and early evolution of star clusters assuming that they form from a turbulent starless clump of given mass bounded inside a parent self-gravitating molecular cloud characterized by a particular mass surface density. As a first step we assume instantaneous star cluster formation and gas expulsion. We draw our initial conditions from observed properties of starless clumps. We follow the early evolution of the clusters up to $20 \mathrm{Myr}$, investigating effects of different star formation efficiencies, primordial binary fractions and eccentricities and primordial mass segregation levels. We investigate clumps with initial masses of $M_{\mathrm{cl}}=3000 \mathrm{M}_{\odot}$ embedded in ambient cloud environments with mass surface densities, $\Sigma_{\text {cloud }}=0.1$ and $1 \mathrm{~g} \mathrm{~cm}^{-2}$. We show that these models of fast star cluster formation result, in the fiducial case, in clusters that expand rapidly, even considering only the bound members. Clusters formed from higher $\Sigma_{\text {cloud }}$ environments tend to expand more quickly, so are soon larger than clusters born from lower $\Sigma_{\text {cloud }}$ conditions. To form a young cluster of a given age, stellar mass and mass surface density, these models need to assume a parent molecular clump that is many times denser, which is unrealistic compared to observed systems. We also show that in these models the initial binary properties are only slightly modified by interactions, meaning that binary properties, e.g., at $20 \mathrm{Myr}$, are very similar to those at birth. With this study we set up the basis of future work where we will investigate more realistic models of star formation compared to this instantaneous, baseline case.
\end{abstract}

Keywords: methods: numerical — galaxies: star formation, star clusters

\section{INTRODUCTION}

Most stars tend to form together in clusters (e.g., Gutermuth et al. 2009), which are created from over dense gas clumps, typically found in giant molecular clouds (GMCs) (e.g., McKee \& Ostriker 2007). Thus understanding how stars form comes with the direct need to understand how and where star clusters form. It is still a matter of debate if star cluster formation depends on properties of the GMC environment or not. While theoretical studies have taught us the essential physical processes that determine a star cluster's evolution after gas is dispersed, the transition from the dense star-forming clump to the star cluster that emerges from the gas is not yet well understood (see, e.g., Banerjee \& Kroupa 2015, for a review). In particular, it is debated whether the process is slow with the clump evolving in a quasiequilibrium state (Tan et al. 2006; Nakamura \& Li 2007), or very rapid with star cluster formation occurring in just a crossing time of the system (Elmegreen 2000, 2007; Hartmann \& Burkert 2007).

There are numerous physical processes potentially involved in such a transition from gas clump to star cluster, including: fragmentation of a magnetized, turbulent and self-gravitating medium to a population of pre-stellar cores; collapse of these cores via rotationally supported disks to single or multiple star systems; feedback from the forming stars, especially protostellar outflows that can maintain turbulence in the clump (Nakamura \& Li 2007,2014 ), and eventually radiative feedback processes

^E-mail:jfarias@ufl.edu from more massive stars (e.g., Dale et al. 2015); continued infall of gas to the clump; dispersal of clump gas by feedback; dynamical interactions among the forming and recently formed stars as they orbit in the protocluster potential (e.g., Chatterjee \& Tan 2012). This is a complicated, multi-scale problem, the full solution of which is beyond current computational capabilities. Thus approximate models are necessary. By investigating how the outcome of star cluster formation depends on the adopted approximations we can learn which processes are most important.

Our approach in this paper and subsequent papers in this series is to follow the dynamics of formed stars, including binary properties, accurately via direct $N$ body integrations, but approximate various models for how individual stars are born within the star-forming clump. Our initial conditions are based on the Turbulent Core/Clump model of McKee \& Tan (2003) (hereafter MT03), which approximates clumps as singular polytropic virialized spheres that are in pressure equilibrium with their surrounding cloud medium. This surrounding cloud is also assumed to be self-gravitating so its ambient pressure is $\bar{P} \sim G \Sigma^{2}$, where $\Sigma$ is the cloud mass surface density - the main variable describing different environmental conditions.

In this first paper, we start with the simplest approximation for star formation, i.e., instantaneous formation of the stellar population from the initial gas clump along with simultaneous, instantaneous expulsion of the remaining gas that is not incorporated into stars. This approximation has often been adopted by previous $N$ - 
body studies (e.g., Bastian \& Goodwin 2006; Parker et al. 2014; Pfalzner et al. 2015). However, in comparison to these previous studies our work is distinguished by (1) adopting initial conditions that have been explicitly developed for self-gravitating gas clumps (i.e., singular polytropic spheres as approximations for turbulent, magnetized clumps); (2) following the full evolution of binary systems.

A number of authors have studied the dynamics of binaries in star clusters using numerical models (e.g., Kroupa et al. 1999; Kroupa \& Burkert 2001; Parker et al. 2009; Kouwenhoven et al. 2010; Parker et al. 2011; Kaczmarek et al. 2011), focusing on various aspects of their dynamics. In our work we follow the evolution of binary properties due to stellar interactions and stellar evolution in clusters forming in different environments. We use and will use these results to constrain assumptions about the star cluster process, e.g., its duration, and individual star formation processes, e.g., how binaries form, i.e., the primordial binary properties. We also examine the role of binaries in producing dynamical and binary supernova ejections, especially fast runaway stars.

We anticipate that, in reality, star cluster formation is likely to proceed in a relatively gradual manner, i.e., taking at least several and perhaps many local free-fall timescales of the gas clump. Modeling this process of gradual star cluster formation will be the topic of the second paper in this series. One aspect that is needed in such a model is the gradual evolution of the potential of the natal gas clump, which can be approximated via a simple analytic relation. Such an approach of an evolving approximate background gas potential has been adopted previously by (e.g., Tutukov 1978; Lada et al. 1984; Geyer \& Burkert 2001; Boily \& Kroupa 2003; Chen \& Ko 2009; Smith et al. 2011; Farias et al. 2015; Brinkmann et al. 2016). However, in all these studies the full stellar population was introduced instantaneously at the beginning of the simulation. None of these studies included a full treatment of binaries, i.e., including a significant fraction of primordial binaries.

The second aspect is the gradual formation of the stellar population in the cluster. This will be the main focus of Paper II in our series. There have so far been relatively few studies using such an approach. Proszkow \& Adams (2009) presented a study involving gradual star formation and then the early evolution of young clusters, focusing on low to intermediate mass clusters (100 to 3000 members), without primordial binaries. However, the stellar densities of their models were relatively low, limiting the effects of stellar interactions. They also did not include stellar evolution and only simulated up to $10 \mathrm{Myr}$.

These methods of $N$-body modeling can be contrasted with other approaches to simulating star cluster formation, e.g., simulations that follow the (magnetohydrodynamics of the collapsing clump (e.g., Price \& Bate 2009; Padoan et al. 2012; Myers et al. 2014; Padoan et al. 2014). Such simulations must still implement subgrid models for how stars form and inject feedback into the gas. Typically they do not have the resolution to accurately follow binary orbital evolution. Still, these are complementary approaches to those based on pure $N$-body approaches and comparison of the results of the different methods will be instructive.

\section{PRE-CLUSTER CLUMPS AS INITIAL CONDITIONS}

The initial conditions for star clusters are constrained by the observed properties of dense gas clumps within GMCs. These locations are also expected to be the sites of future massive star formation. The properties of these clumps have been summarized by Tan et al. (2014), based on Galactic observational studies of Infrared Dark Clouds (IRDCs) (e.g., Rathborne et al. 2006; Butler \& Tan 2009, 2012) and mm/sub-mm dust continuum emission and molecular line surveys of clumps (e.g., Schuller et al. 2009; Ginsburg et al. 2012; Ma et al. 2013). There are a range of clump masses observed from $\sim 100 M_{\odot}$ to $\sim 10^{5} M_{\odot}$.

In the fiducial Turbulent Clump model, the mass surface density of the clump of interest is only a factor of 1.22 times higher than that of its surrounding cloud (MT03). Therefore the observed surface densities of clumps give a reasonable estimate of the mass surface densities of the ambient clouds, $\Sigma_{\text {cloud }}$, which is the other main parameter needed to set up the initial conditions of the models. The observed values of mass surface density of Galactic clumps and protoclusters are in the range from $\sim 0.03-1 \mathrm{~g} \mathrm{~cm}^{-2}$.

In the setup of our initial conditions in this paper we make several simplifying assumptions as first steps in describing the complexity of star cluster formation: i) the parent clump is isolated (i.e., no external tidal fields); ii) the clump is in hydrostatic and virial equilibrium with the structure of a singular polytropic sphere (MT03); iii) stars are born with the same velocities as their parent gas, so that their velocity dispersion profile is the same as that of the initial gas; iv) all stars form instantaneously and the remaining gas is also expelled instantaneously at this time; iv) the star formation efficiency (SFE) is spatially constant, which means that stars follow the same spatial distribution as the initial gas; v) there is no initial spatial or kinematic substructure given to the stars, except that which results from random, Poisson sampling; vi) following an initial test model of equal mass stars, a standard Kroupa IMF for the stars (Kroupa 2001) is adopted, then with various binary properties investigated. It should be remembered that these are starting assumptions and that many of these will be relaxed in subsequent investigations. However, first the behavior of this simplest, idealized model needs to be understood.

\subsection{Initial stellar phase-space distributions}

First we define the physical and kinematic properties of the pre-cluster clump, i.e., mass, size, density profile and velocity dispersion profile. Stars are born from this clump and initially follow the same phase-space (position, velocity) distribution. MT03 characterize precluster clumps and pre-stellar cores as singular polytropic spheres in virial and hydrostatic equilibrium. The density profile of such clumps is then:

$$
\rho_{\mathrm{cl}}(r)=\rho_{\mathrm{s}, \mathrm{cl}}\left(\frac{r}{R_{\mathrm{cl}}}\right)^{-k_{\rho}},
$$

where $\rho_{\mathrm{s}, \mathrm{cl}}$ is the density at the surface of the clump, i.e., at radius $R_{\mathrm{cl}}$. We adopt $k_{\rho}=1.5$ as a fiducial value, i.e., the same as that of MT03 who made their choice based on observations of clumps reported at the time. 
No significant difference has been found in later measurements performed by Butler \& Tan (2012) in IRDCs where they found $k_{\rho} \simeq 1.6$. We thus keep $k_{\rho}=1.5$ as our fiducial value for simplicity and consistency with the previous analysis of MT03. The density at the surface of the clump can be expressed as

$$
\rho_{\mathrm{s}, \mathrm{cl}}=\frac{\left(3-k_{\rho}\right) M_{\mathrm{cl}}}{4 \pi R_{\mathrm{cl}}^{3}}
$$

where $M_{\mathrm{cl}}=M\left(r<R_{\mathrm{cl}}\right)$ is the total mass of the clump.

The radius of a clump in virial equilibrium and pressure equilibrium with its surroundings, i.e., a larger selfgravitating cloud of given mass surface density, $\Sigma_{\text {cloud }}$, is (MT03; Tan et al. 2013, hereafter T13)

$$
\begin{aligned}
R_{\mathrm{cl}}= & 0.50\left(\frac{A}{k_{p} \phi_{P, \mathrm{cl}} \phi_{\bar{P}}}\right)^{1 / 4}\left(\frac{M_{\mathrm{cl}}}{3000 \mathrm{M}_{\odot}}\right)^{1 / 2} \\
& \times\left(\frac{\Sigma_{\text {cloud }}}{1 \mathrm{~g} \mathrm{~cm}^{-2}}\right)^{-1 / 2} \mathrm{pc} \\
\rightarrow & 0.37 M_{\mathrm{cl}, 3000}^{1 / 2} \Sigma_{\text {cloud }, 1}^{-1 / 2} \mathrm{pc}
\end{aligned}
$$

where $k_{p}=2\left(k_{\rho}-1\right)$ is the power law exponent of the pressure $(P)$ within the clump; $\phi_{P, c l}$ is the ratio between the pressure at the surface of the clump $\left(P_{\mathrm{s}, \mathrm{cl}}\right)$ and the mean pressure inside the cloud, $\bar{P}_{\text {cloud }} ; \phi_{\bar{P}}$ is a normalization constant, $\sim \mathcal{O}(1)$, in the relation $P_{\text {cloud }} \equiv$ $\phi_{\bar{P}} G \Sigma_{\text {cloud }}^{2}$; and $A=\left(3-k_{\rho}\right)\left(k_{\rho}-1\right) f_{g} \rightarrow 3 / 4$, since we assume the clump is initially starless so $f_{g}=1$. As fiducial values we choose $\phi_{P, \mathrm{cl}}=2$ and $\phi_{\bar{P}}=1.32$ (see MT03; T13). Thus the structural properties of the fiducial clump are specified by two parameters: $M_{\mathrm{cl}}$ and $\Sigma_{\text {cloud }}$. In this study we will focus on the case of $M_{\mathrm{cl}}=3000 \mathrm{M}_{\odot}$ and investigate $\Sigma_{\text {cloud }}=0.1$ and $1 \mathrm{~g} \mathrm{~cm}^{-2}$, which are representative of the range of values observed in real clumps (Tan et al. 2014).

The total mass of stars, $M_{*}$, that form from the clump is given

$$
M_{*}=\epsilon M_{\mathrm{cl}},
$$

where $\epsilon$ is the overall SFE. We will consider a range of values from $\epsilon=0.1$ to 1 , with a fiducial value of 0.5 . The stars are assumed to have the same structural profile as the clump, i.e., $\epsilon$ is independent of radial location. Then, the density profile of the stars is simply: $\rho_{*}(r)=\epsilon \rho_{\mathrm{cl}}(r)$. The cumulative radial mass distribution of the stars is

$$
M_{*}\left(r<R_{\mathrm{cl}}\right)=M_{*}\left(\frac{r}{R_{\mathrm{cl}}}\right)^{3-k_{\rho}} .
$$

To set up the positions of the stars, we first create the mass sample from a given IMF (including binary companions), i.e., in the fiducial case a standard Kroupa IMF (Kroupa 2001) with individual masses in the range $0.01 \mathrm{M}_{\odot}<m_{i}<100 \mathrm{M}_{\odot}$, in random order. Next, we create a cumulative mass array from the previous sample to then choose the individual distance from the center $r_{i}$ according to Eq. 6 (in the case of $100 \%$ mass segregation, see below, masses are sorted from the most massive to the less massive before this step). Finally, we place the star randomly on the surface of the sphere of radius $r_{i}$. In this way we ensure that the clusters always match the desired initial density profile, no matter the nature of the stellar IMF.

The kinematic properties of the clump are specified by the condition of virial equilibrium. In a virialized clump, the velocity dispersion $\sigma$ scales in the same manner as the effective sound speed $c \equiv(P / \rho)^{1 / 2}$. Therefore, the velocity dispersion profile of the clump is:

$$
\sigma_{\mathrm{cl}}(r)=\sigma_{\mathrm{cl}, \mathrm{s}}\left(\frac{r}{R_{\mathrm{cl}}}\right)^{\left(2-k_{\rho}\right) / 2},
$$

where $\sigma_{\mathrm{cl}, \mathrm{s}}$ is the velocity dispersion at the surface of the clump. The presence of large-scale magnetic fields can provide some support to the clump so that a smaller turbulent velocity dispersion is needed to achieve virial equilibrium. The effect of magnetic fields on the stability of the clump can be expressed as $\phi_{\mathrm{B}} \equiv\left\langle c^{2}\right\rangle /\left\langle\sigma^{2}\right\rangle$. Then, the velocity dispersion at the surface is:

$$
\begin{aligned}
\sigma_{\mathrm{cl}, \mathrm{s}}= & 5.08\left(\frac{\phi_{P, \mathrm{cl}} \phi_{\bar{P}}}{A k_{P}^{2} \phi_{\mathrm{B}}^{4}}\right)^{1 / 8}\left(\frac{M_{\mathrm{cl}}}{3000 \mathrm{M}_{\odot}}\right)^{1 / 4} \\
& \times\left(\frac{\Sigma_{\text {cloud }}}{1 \mathrm{~g} \mathrm{~cm}^{-2}}\right)^{1 / 4} \mathrm{~km} \mathrm{~s}^{-1}
\end{aligned}
$$

where we use $\phi_{\mathrm{B}}=2.8$ as a fiducial value, which is the value for regions with an Alfvén Mach number of 1 (see Appendix A2 of MT03). The initial velocity dispersion profile of the stars then follows Eq. 7. The individual stellar velocities are then assigned velocities in each of the $x, y$ and $z$ directions independently by drawing from a Gaussian centered at zero with width $\sigma(r)$. Note that the mass averaged velocity dispersion of the clump/cluster is (T13)

$$
\sigma_{\mathrm{cl}}=\frac{2\left(3-k_{\rho}\right)}{8-3 k_{\rho}} \sigma_{\mathrm{cl}, \mathrm{s}} \rightarrow \frac{6}{7} \sigma_{\mathrm{cl}, \mathrm{s}}
$$

The resulting velocity distribution has the form of a Maxwell-Boltzmann distribution with $\sigma_{3 D}=\sqrt{3} \sigma_{\mathrm{cl}}$ but with a one dimensional velocity dispersion profile as in equation 7 . The properties of the clumps, i.e., the low and high $\Sigma$ cases, are summarized in Table 1.

\subsection{The primordial binary population}

Observational evidence shows that about half of star systems in the field are binaries (e.g., Duquennoy \& Mayor 1991; Fischer \& Marcy 1992; Mason et al. 1998; Preibisch et al. 1999; Close et al. 2003; Basri \& Reiners 2006; Raghavan et al. 2010). Given the densities of starforming clumps and young star clusters, it is likely that most of these binaries where born together inside individual cores, rather then forming via subsequent dynamical interactions (e.g., Parker \& Meyer 2014). However, this is a question that our simulations will be able to address.

Theoretically, a full understanding of binary formation from a collapsing gas core is likely to require a full nonideal MHD treatment to resolve formation of the accretion disk and then later its potential fragmentation. The difficulty of this problem means that essentially the statistical properties of primordial binaries are very uncertain, and so we will investigate several different choices based on observations.

For most of our simulations that include binaries, we assume a binary system fraction, $f_{\text {bin }}=0.5$. We adopt 
Table 1

Parent clump parameters

\begin{tabular}{ccccccccc}
\hline & $\Sigma_{\text {cloud }}$ & $M_{\mathrm{cl}}\left(\mathrm{M}_{\odot}\right)$ & $R_{\mathrm{cl}}(\mathrm{pc})$ & $k_{\rho}$ & $\phi_{P, \mathrm{cl}}$ & $\phi_{\bar{P}}$ & $\phi_{\mathrm{B}}$ & $\sigma_{\mathrm{cl}}(\mathrm{km} / \mathrm{s})$ \\
\hline "Low- $\Sigma$ " Clump & 0.1 & 3000 & 1.159 & 1.5 & 2 & 1.31 & 2.8 & 1.71 \\
"High- $\Sigma$ " Clump & 1 & 3000 & 0.367 & 1.5 & 2 & 1.31 & 2.8 & 3.04 \\
\hline
\end{tabular}

Table 2

Initial conditions for simulation sets

\begin{tabular}{rccccccll}
\hline Set name & $\epsilon$ & $\left\langle N_{*}\right\rangle$ & $f_{\text {bin }}$ & $f(e)$ & IMF & IMS & S.E. & Comment in plots \\
\hline equal_mass & 0.5 & 1500 & 0 & - & single mass & N & N & Single equal mass particles \\
single_imf & 0.5 & 4000 & 0 & - & Kroupa (2001) & N & N & Single stars with IMF (No SE) \\
binaries_50 & 0.5 & 4000 & 0.5 & $\delta(e)$ & Kroupa (2001) & N & N & $50 \%$ binaries (No SE) \\
fiducial & 0.5 & 4000 & 0.5 & $\delta(e)$ & Kroupa (2001) & N & Y & Fiducial Case \\
binaries_un & 0.5 & 4000 & 0.5 & uniform & Kroupa (2001) & N & Y & $e$ uniform distribution \\
binaries_th & 0.5 & 4000 & 0.5 & $2 e$ & Kroupa (2001) & N & Y & $e$ thermal distribution \\
segregated & 0.5 & 4000 & 0.5 & $\delta(e)$ & Kroupa (2001) & Y & Y & Mass segregated \\
binaries_100 & 0.5 & 4000 & 1 & $\delta(e)$ & Kroupa (2001) & N & Y & $100 \%$ binaries \\
sfe_10 & 0.1 & 850 & 0.5 & $\delta(e)$ & Kroupa (2001) & N & Y & SFE =10\% \\
sfe_30 & 0.3 & 2500 & 0.5 & $\delta(e)$ & Kroupa (2001) & N & Y & SFE $=30 \%$ \\
sfe_80 & 0.8 & 6500 & 0.5 & $\delta(e)$ & Kroupa (2001) & N & Y & SFE $=80 \%$ \\
sfe_100 & 1.0 & 7300 & 0.5 & $\delta(e)$ & Kroupa (2001) & N & Y & SFE $=100 \%$ \\
\hline
\end{tabular}

Note. - For each of the sets named in the first column 20 simulations were performed for each of the clumps parameters listed in Table 1. Second column shows the assumed SFE, third column shows the average number of stars per simulation, fourth column the primordial binary fraction, fifth column the eccentricity distribution function, column six shows the assumed IMF, column seven stands for whether the cluster is initially mass segregated (IMS). Column eight shows if Stellar evolution is included in the set and last column is a comment from which we will referring to the set in the graphs for clarity.

a period distribution from the survey (Raghavan et al. 2010) using a log-normal period distribution with mean of $P=293.3$ years and standard deviation of $\sigma_{\log P}=$ 2.28 (with $P$ in days). We use a companion mass ratio distribution (CMRD) of the form $d N / d q \propto q^{0.7}$, based on observations in young star clusters (Reggiani \& Meyer 2011). The eccentricity distribution remains less well constrained. While Duquennoy \& Mayor (1991) found a thermal distribution, i.e., $f(e)=2 e$ for solar-type stars in the solar neighborhood, a similar more recent study (Raghavan et al. 2010) found a flat eccentricity distribution for the same kind of stars. However, if binaries form mainly via disk fragmentation we would expect that they are born with near circular orbits. In order to measure how much binaries are affected by dynamical interactions in the different models we adopt initially circular orbits for the eccentricities in our fiducial model. We also investigate cases with initially thermal and uniform distributions of eccentricities.

\subsection{Overview of the Cluster Models}

For each of the low and high $\Sigma$ clumps (see Table 1), we set up a stellar cluster as described above, i.e., assuming constant $\operatorname{SFE}(r)$ and a velocity dispersion profile equal to that of the parent clump. Thus, the initial crossing time (i.e., dynamical time) is defined by the properties of the parent clump to be $t_{\mathrm{cr}} \equiv R_{\mathrm{cl}} / \sigma_{\mathrm{cl}}$, i.e., 0.663 and 0.118 Myr for the $\Sigma_{\text {cloud }}=0.1$ and $1 \mathrm{~g} \mathrm{~cm}^{-2}$ cases, respectively.

We run 20 realizations for each set of initial conditions, summarized in Table 2. The simulations are run for 20 Myr, varying only the random seed between simulations in the same set, which affects the initial positions and velocities of the stars as well as the IMF sampling.

We construct these sets of simulations starting from the simplest case to the one that defines our fiducial case.
We start using only single mass particles of $m_{i}=1 \mathrm{M}_{\odot}$ with no primordial binaries and with a SFE of $50 \%$ in the set equal mass. Next, we include an IMF assuming the Kroupa (2001) distribution with a mass range from 0.01 to $100 \mathrm{M}_{\odot}$ defining the set single_imf, again with no initial binaries. We then include $50 \%$ primordial binaries with circular orbits and with other properties described in $\$ 2.2$, defining the set binaries_50. The above three simulation sets do not include stellar evolution (SE). We define the fiducial simulation set by assuming a Kroupa IMF, SFE of $50 \%$, with $50 \%$ of stars as primordial binary systems with initial circular orbits and with stellar evolution included.

Next, we test other choices and parameters of the fiducial model. We test two other eccentricity distributions, a thermal eccentricity distribution, i.e., $f(e)=2 e$ in the set binaries_th, and a uniform eccentricity distribution between 0 and 1 in the set binaries_un. An extreme scenario of mass segregation is tested in the set segregated in which stars are sorted in descending order of individual stellar mass from the center of the cluster. We also test the extreme case in which all stars are binary systems $\left(f_{\text {bin }}=1\right)$ in the set binaries_100.

We also carry out simulations with different SFE. These simulations only differ from the fiducial set in their SFE, i.e., the average number of stars per simulation on each set increases with the SFE since we use the same parent clump of $M_{\mathrm{cl}}=3000 \mathrm{M}_{\odot}$. The SFEs investigated are $\mathrm{SFE}=10 \%, 30 \%, 80 \%$ and $100 \%$ and the sets are named sfe_10, sfe_30, sfe_80 and sfe_100, respectively.

\subsection{Numerical Methods}

We follow the evolution of the star clusters for 20 Myr utilizing the direct $N$-body integrator NBODy $6++$ (Wang et al. 2015) which is a GPU/MPI optimized ver- 


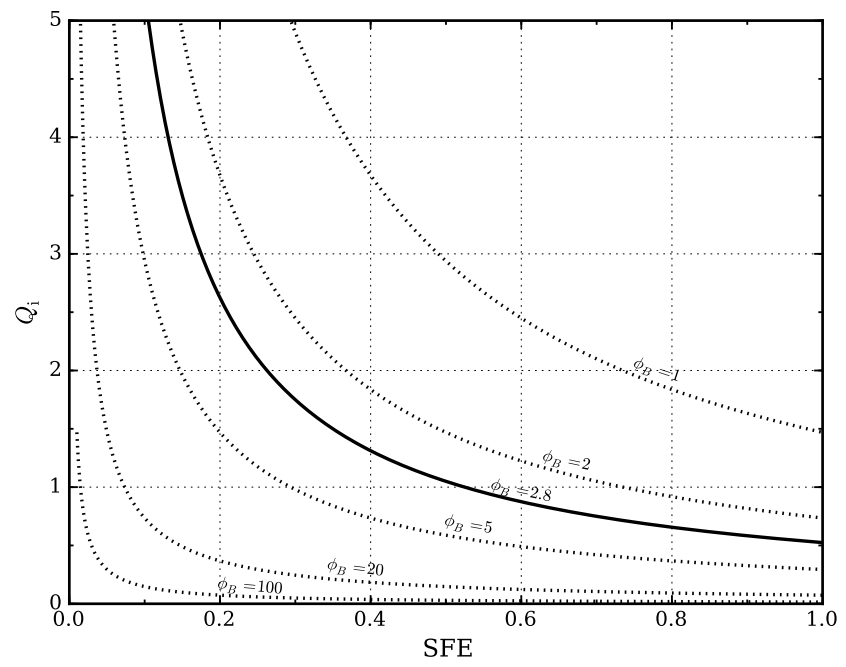

Figure 1. The initial virial ratio $Q_{i}$ as a function of the SFE from the clump. Solid line shows the relation for our fiducial value and dotted lines the relation for different values of $\phi_{\mathrm{B}}$.

sion of the classical and widely used direct integrator NBODy6 (Aarseth 2003). NBODy6++ has implemented special regularizations to accurately follow the evolution of binaries and high order systems in the cluster being able to efficiently simulate star clusters with high binary fractions with no loose of accuracy. For cases with stellar evolution we used the recipe included in NBODY6++ based on the analytical models for single and binary stellar evolution developed by Hurley et al. (2000, 2002). The code also has implemented artificial velocity kicks to emulate asymmetrical supernovae ejections. The magnitude of the kicks are drawn from a Maxwell distribution with $\sigma=265 \mathrm{~km} / \mathrm{s}$ following the observations of Hobbs et al. (2005) on pulsar proper motions.

\section{RESULTS}

\subsection{Initial dynamical state of the clusters}

Before performing any simulation, from the assumptions described in $\S 2$, we first derive the initial dynamical state of the clusters by characterizing their virial ratio, i.e.,

$$
Q_{i}=-\frac{T_{*}}{\Omega}
$$

where $T_{*}$ is the total kinetic energy of the stars and $\Omega$ is their total gravitational potential energy. A cluster with $Q_{i}<1$ is bound and $Q_{i}=0.5$ is the value for a state of virial equilibrium. We assumed the gas was expelled immediately after the stars formed, thus $\Omega$ only depends on the stars in the cluster, i.e., $\Omega=\Omega_{*}$. The potential of the stars is then

$$
\begin{aligned}
\Omega_{*}= & -\frac{G}{2} \int_{0}^{R_{\mathrm{cl}}}\left[\frac{M\left(r<R_{\mathrm{cl}}\right)}{r}\right]^{2} \mathrm{~d} r \\
& -\frac{G}{2} \int_{R_{\mathrm{cl}}}^{\infty}\left(\frac{M_{*}}{r}\right)^{2} \mathrm{~d} r \\
= & -\left(\frac{3-k_{\rho}}{5-2 k_{\rho}}\right) \frac{G M_{*}^{2}}{R_{\mathrm{cl}}} .
\end{aligned}
$$

The kinetic energy of the stars is given by

$$
T_{*}=\frac{3}{2} M_{*} \sigma^{2},
$$

where $\sigma$ is the one dimensional mass averaged velocity dispersion. Assuming that the stars are born from the gas following the same dispersion profile, then $\sigma$ is related to that at the surface by Eq. 9 .

Replacing equations 9, 13 and 12 in eq. 10, and also replacing $R_{\mathrm{cl}}$ and $\sigma_{\mathrm{cl}, \mathrm{s}}$ by their expressions in eq. 4 and 8 respectively, we obtain

$$
\begin{aligned}
Q_{i} & =\frac{3\left(5-2 k_{\rho}\right)\left(3-k_{\rho}\right)}{\left(8-3 k_{\rho}\right)^{2}\left(k_{\rho}-1\right)} \frac{1}{\epsilon \phi_{\mathrm{B}}} \\
Q_{i} & \rightarrow \frac{0.51}{\epsilon},
\end{aligned}
$$

where the arrow shows the relation using the fiducial values for the clump. Values of $Q_{i}$ versus SFE are shown for different models in Figure 1.

The dynamical state of the clusters also depends on the presence of magnetic fields in the initial clump, i.e., $\phi_{B}$. In the absence of magnetic field support $\left(\phi_{B}=1\right)$ the velocities needed for virial equilibrium are higher and stars formed from such gas will have higher values of $Q_{i}$, e.g., even in the best case scenario with a $\mathrm{SFE}$ of a $100 \%$ we have $Q_{i} \simeq 1.6$ (and $\simeq 3$ for $\mathrm{SFE}$ of $50 \%$ ). However, in the fiducial case with approximate equipartition of energy density from large scale magnetic fields and turbulence $\left(\phi_{B} \simeq 2.8\right)$ a $\mathrm{SFE}$ of about $50 \%$ leads to a cluster that is marginally gravitationally bound $\left(Q_{i} \simeq 1\right)$. Note that this variation of $\phi_{B}$ also corresponds to a variation in the virial parameter of the gas clump, $\alpha_{\mathrm{vir}}=5\left\langle\sigma^{2}\right\rangle R /(G M)$, since for the fiducial case with $k_{\rho}=1.5$ we have $\alpha_{\text {vir }}=15 /\left(4 \phi_{B}\right) \rightarrow 1.34$ (see Appendix A of MT03).

\subsection{The bound stellar cluster}

As discussed in $\S 3.1$, star clusters born from turbulent clumps bounded by high pressure ambient environments can start with relatively high velocity dispersions. Their virial ratio after gas expulsion will depend on the global SFE and the contribution of magnetic fields to the support of the parent clump, i.e $\phi_{\mathrm{B}}$. There will be significant initial mass loss of the stars that are born unbound, occurring on a timescale of a few crossing times. However, the gaseous clump is assumed to have a positive power law for the velocity dispersion with radius (see Eq. 9) and so is more likely to be left with a central gravitationally bound core. In contrast, a relaxed star cluster (e.g., with a Plummer profile) has a velocity dispersion profile that decreases with radius. We thus expect differences in the early evolution of our clusters compared to those modeled with initial Plummer profiles by, e.g. Goodwin \& Bastian (2006); Baumgardt \& Kroupa (2007); Pfalzner \& Kaczmarek (2013).

To measure the bound mass fraction, $f_{\text {bound }}$, at each timestep of cluster evolution. We construct the bound entity based on an accurate measure of the mean velocity of the bound stars and we select all stars with negative total energy in the frame of reference of the bound cluster. The velocity of the bound cluster is not known $a$ priori (although it is expected to be close to the zero velocity of the reference frame), and thus this is solved in an 


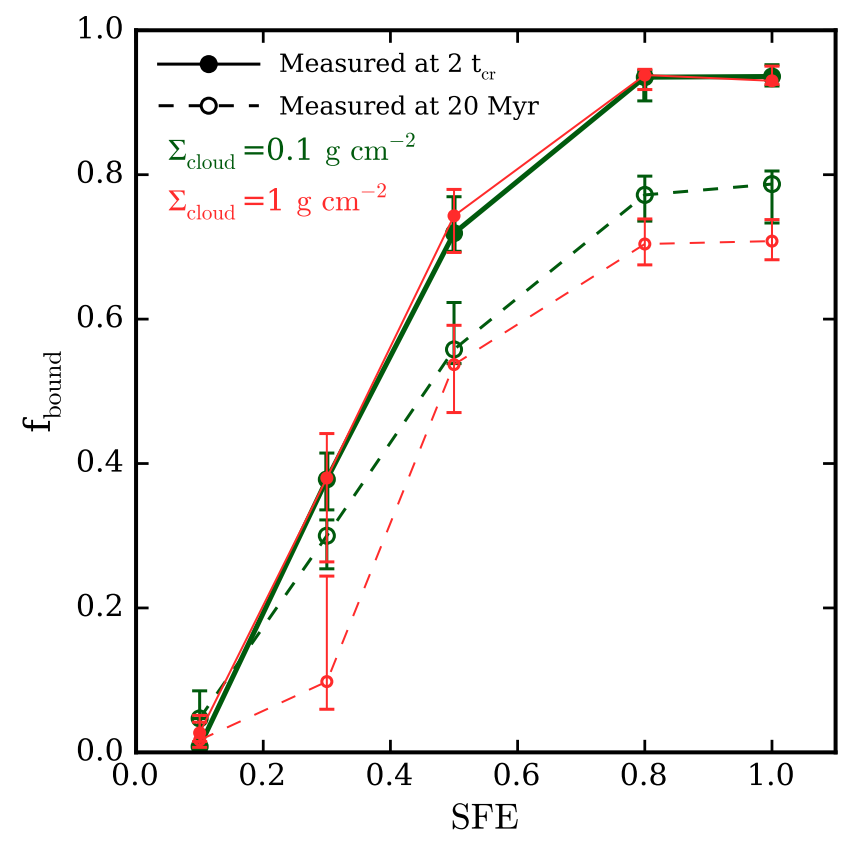

Figure 2. Bound mass fraction, $f_{\text {bound }}$, as a function of SFE. Solid lines and points show results at early times $\left(2 t_{\mathrm{cr}}\right)$; dashed lines and open points show results at the end of the simulations (20 Myr). The values are the mean averages for the simulation sets (each of 20 realizations) with cases shown for $\Sigma_{\text {cloud }}=0.1$ (green) and $1 \mathrm{~g} \mathrm{~cm}^{-2}$ (red). Errorbars show the values between the 25 th and 75 th percentiles. Dynamical evolution leads to a general decrease of $f_{\text {bound }}$ during the first 20 Myrs of these clusters.

iterative calculation. We start by selecting all stars with negative energy inside the half mass radius of the full cluster and then iterate until the members between iterations do not change by more than two members. This method, called "snowballing", is described in Smith et al. (2013b).

Figure 2 shows the bound fractions measured at 2 crossing times and after 20 Myr for clusters with different SFE for simulations with a parent clump with $\Sigma_{\text {cloud }}=0.1$ and $1.0 \mathrm{~g} \mathrm{~cm}^{-2}$. The initially positive radial gradient of velocity dispersion of the star clusters causes stars in the outer parts to leave first, while the central core can remain bound. The mass fraction of this remnant bound core depends on the initial global SFE. Later dynamical evolution and internal mass loss of its members due to stellar evolution leads to a slower decrease in mass of the bound core over time.

The results shown in Figure 2 are those for the fiducial case, i.e., with $\phi_{\mathrm{B}}=2.8$. As discussed in $\S 3.1$, the initial virial ratio of the star clusters depends sensitively on this value: a higher value of $\phi_{\mathrm{B}}$ shifts the trend shown in this figure upwards so that even clusters with $\mathrm{SFE}=10 \%$ may retain a significant bound core if $\phi_{\mathrm{B}}$ is sufficiently high.

\subsection{Global evolution}

Here we explore the evolution of the structure of the clusters with time. The fiducial case has SFE of $50 \%$ and $Q_{i}=1.02$, slightly above the criterion for global boundedness. Therefore, initial expansion and some initial mass loss is expected. We show the evolution of the Lagrangian radii with time in Figures 3 and 4 for $\Sigma_{\text {cloud }}=0.1$ and $1 \mathrm{~g} \mathrm{~cm}^{-2}$, respectively, where the values presented are the average of the 20 simulations performed for each set. In each figure, the top four panels, (a) to (d), show the effects of gradually adding greater degrees of realism to make the fiducial model. Then the lower four panels show the effects of different choices of initial binary properties and degree of initial mass segregation. These figures also show the evolution of the core radius $r_{\mathrm{c}}$ (red dashed line), which is the density averaged distance from the density center of the cluster (see $§ 15.2$ in Aarseth 2003).

As expected, the clusters expand with time. The expansion rate of the outer Lagrangian radii of the clusters, i.e., of the unbound stars, is determined by the initial velocity dispersion of the parent clump, which is higher at higher mass surface densities. Thus star clusters starting from a clump with $\Sigma_{\text {cloud }}=1 \mathrm{~g} \mathrm{~cm}^{-2}$ are soon more extended than the clusters forming from clumps with $\Sigma_{\text {cloud }}=0.1 \mathrm{~g} \mathrm{~cm}^{-2}$ of the same mass and SFE, i.e., the half-mass radius at $20 \mathrm{Myr}$ of the first group is $\sim 20 \mathrm{pc}$ compared to $\sim 10 \mathrm{pc}$ for the lower $\Sigma$ case.

Initial expansion of the bound portion of the cluster happens early within a few crossing times as the clusters relax to a virialized state. Then the later evolution is affected by dynamical interactions between the stars (i.e., mass segregation, evolution of binaries and dynamical ejection of stars from unstable multiple systems) and mass loss resulting from stellar evolution. The relative importance of these effects can be gauged by examining the sequence of panels from (a) to (d) in Figures 3 and 4. The later stage expansion of the bound cluster is negligible in the case of equal mass stars. The model with an IMF but only single stars undergoes mass segregation that leads to noticeable expansion after about $6 \mathrm{Myr}$ in the case of $\Sigma_{\text {cloud }}=0.1 \mathrm{~g} \mathrm{~cm}^{-2}$ and after about 1 Myr in the case of $\Sigma_{\text {cloud }}=1.0 \mathrm{~g} \mathrm{~cm}^{-2}$.

Note that before adding binaries and stellar evolution in our models, evolution of the star clusters with high $\Sigma_{\text {cloud }}$ would be exactly the same as those with low $\Sigma_{\text {cloud }}$ after properly scaling for the initial size and crossing time (see Aarseth \& Heggie 1998). However, the characteristic timescales introduced by binaries (e.g., at their typical orbital separation) and by stellar evolution break this self-similarity.

When binaries are added, their presence leads to another potential source of expansion, since their binding energy couples with the internal energy of the stellar cluster, leading to a change of kinetic energy in each interaction (Heggie 1975; Hills 1975). However little difference appears when moving from single stars to $50 \%$ binaries, even in simulations with the high $\Sigma$ initial condition that can suffer more interactions. As we will see in $\S 3.4$, the initial densities of these models are not high enough and/or do not last long enough to give binaries, on average, the chance to interact significantly with other stars.

The inclusion of stellar evolution causes the cluster to expand even more. Stellar evolution starts becoming important after a few Myr, when the first massive stars lose mass by stellar winds and then explode as supernovae. The supernova explosions may cause stars to be ejected (e.g., as fast runaway stars) either by the destruction of tight binaries, velocity kicks caused by the asymmetrical explosion, or both combined effects (see the outer two lines in Figures 3 and 4). We focus on the ejection of 


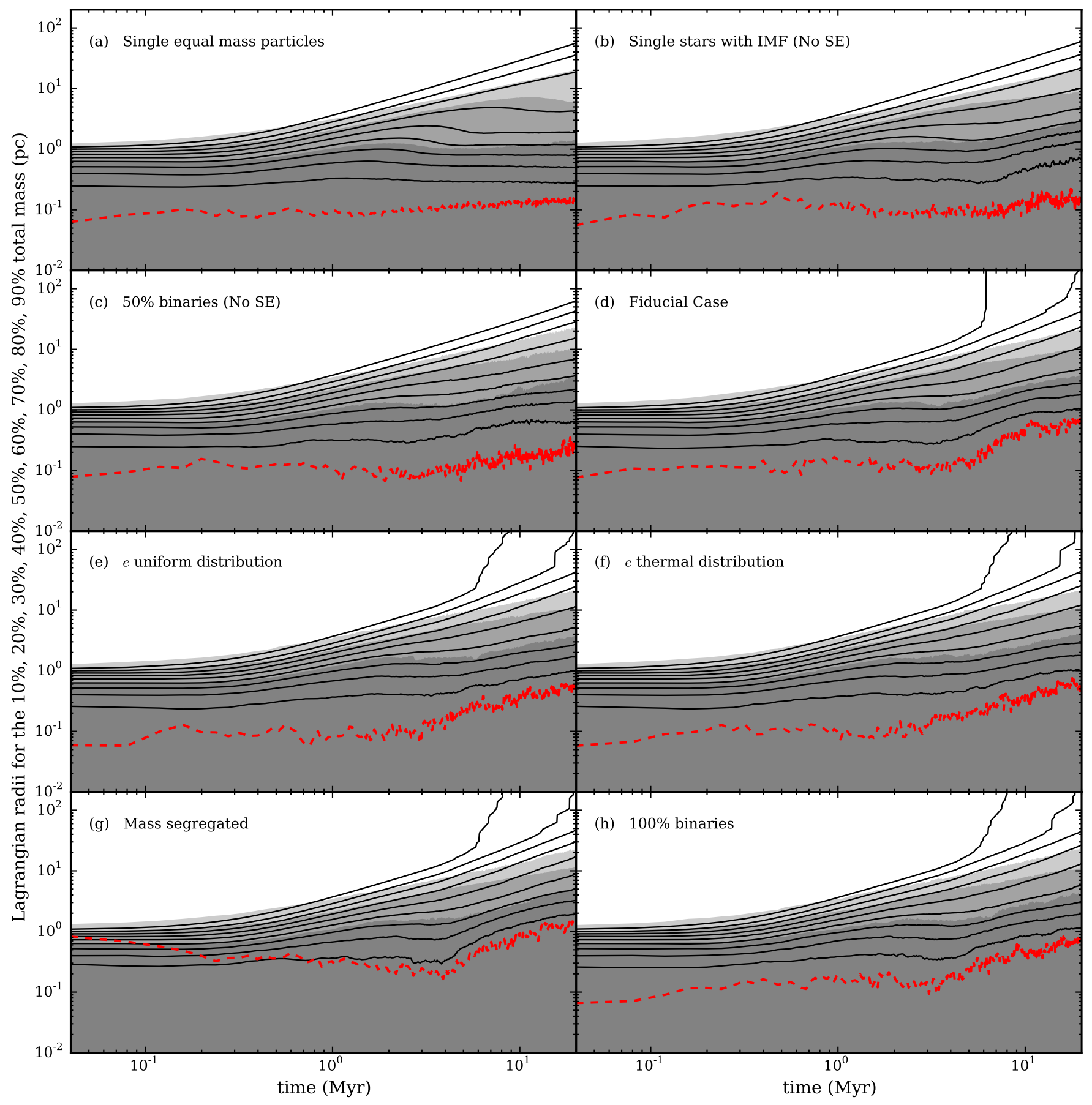

Figure 3. Average Lagrangian radii evolution for different set of simulations for star clusters born from a parent clump with $\Sigma_{\text {cloud }}=$ $0.1 \mathrm{~g} \mathrm{~cm}^{-2}$. We show the Lagrangian radii for the $10 \%, 20 \%, 30 \%, 40 \%, 50 \%, 60 \%, 70 \%, 80 \%$ and $90 \%$ masses (black solid lines). Red dashed lines are the core radii defined in Aarseth (2003). Gray shaded areas represent the regions below the 50\%, 95\% and 100\% mass radius of the bound cluster.

runaway stars below in $\S 3.6$. For now we see that cluster expansion is increased by this effect and also by the fact that the potential well of the cluster is made more shallow with the loss of mass through stellar winds and supernovae. However, the loss of runaway stars does not affect the global evolution of the cluster too much, even in the most extreme case with $f_{\text {bin }}=1$. Finally, the lower four panels in these figures show that variations of binary orbital properties, degree of initial mass segregation or primordial binary fractions have relatively minor effects.

In Figure 5 we compare the evolution of several parameters of the different sets of simulations for clusters born with different initial mass surface densities: $\Sigma_{\text {cloud }}=0.1 \mathrm{~g} \mathrm{~cm}^{-2}$ on the left; $\Sigma_{\text {cloud }}=1 \mathrm{~g} \mathrm{~cm}^{-2}$ on the right. In the first row we show evolution of the bound mass fraction $f_{\text {bound }}$. Only for the purposes of this figure, to show the timescale on which initially unbound stars leave the cluster, we count all stars inside the $95 \%$ radius of the bound cluster as also being bound. Here, 


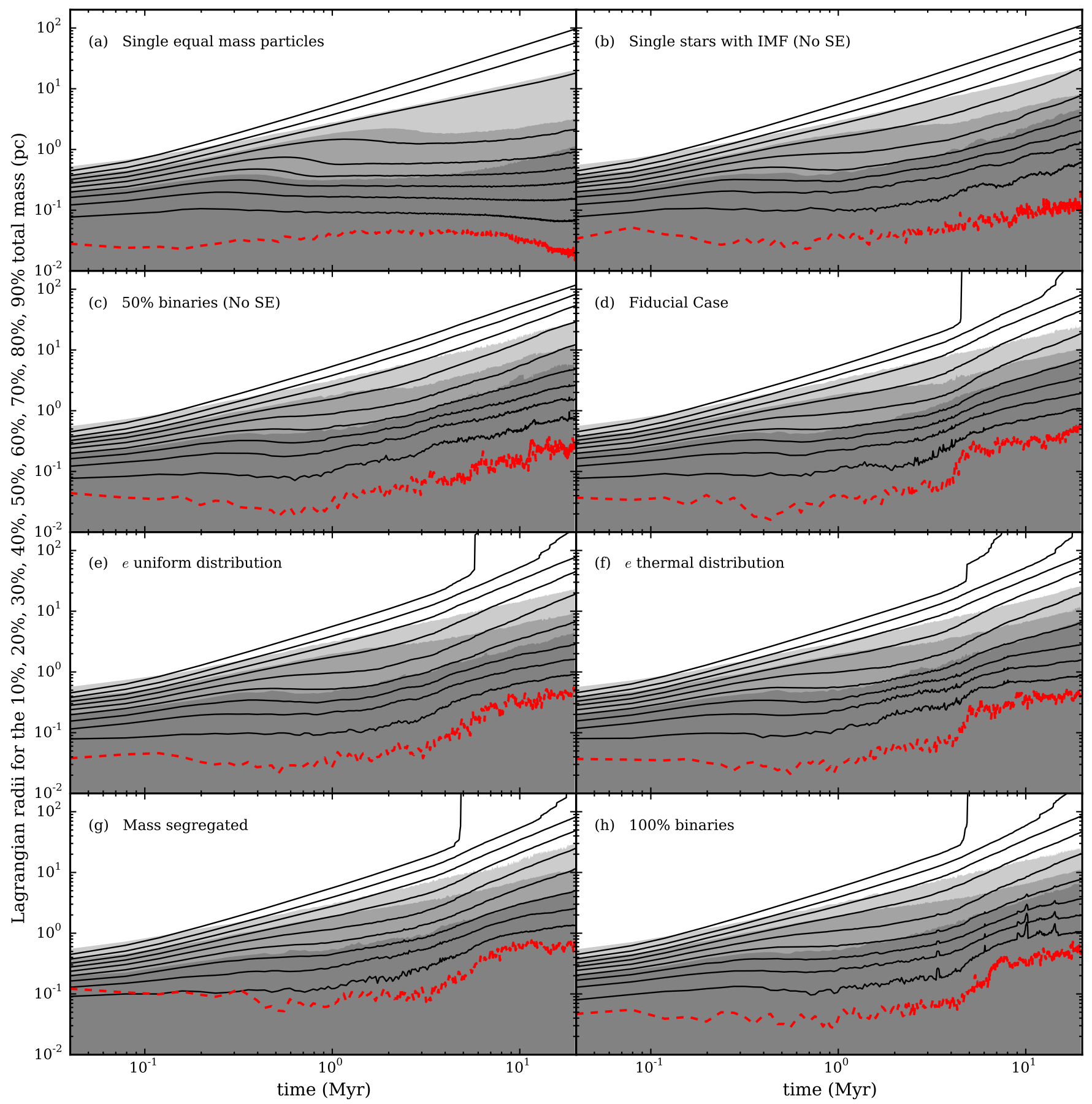

Figure 4. Same as Figure 3 for star clusters born from a clump with $\Sigma_{\text {cloud }}=1 \mathrm{~g} \mathrm{~cm}^{-2}$.

and in all panels in this figure, values are the medians of each set of simulations with parameters given in Table 2. Also shown in the top row is a shaded area representing the loss of mass due to stellar evolution for all stars in the fiducial simulations (including unbound stars). The second row shows the evolution of the core radii, $r_{\mathrm{c}}$, and the half-mass radii, $r_{\mathrm{h}}$. The third row shows the evolution of the effective number density, $n_{\mathrm{s}}$, i.e., the number of systems (a binary is counted as one system) inside the volume defined by $r_{\mathrm{h}}$. The fourth row shows the evolution of the velocity dispersion measured inside $r_{\mathrm{h}}$, while the fifth row shows the evolution of the total binary frac- tion.

It takes about $1.5 t_{\text {cr }}$ for initially unbound stars to leave the bound cluster, leading to $f_{\text {bound }}$ decreasing to about 0.7 . The velocity profile of the clusters have a positive slope, i.e., higher speeds in the outskirts, this causes outer stars to be more likely to be unbound, with practically no chance of interacting with others. These stars leave the cluster with a velocity dispersion determined by the parent clump, i.e. $\sigma_{\mathrm{cl}, \mathrm{s}}$. We refer to these as unbound stars, distinguishing from the ejected stars that escape later due to dynamical ejections. After the first $\sim 1.5 t_{\mathrm{cr}}$, all initially unbound stars leave the cluster and 


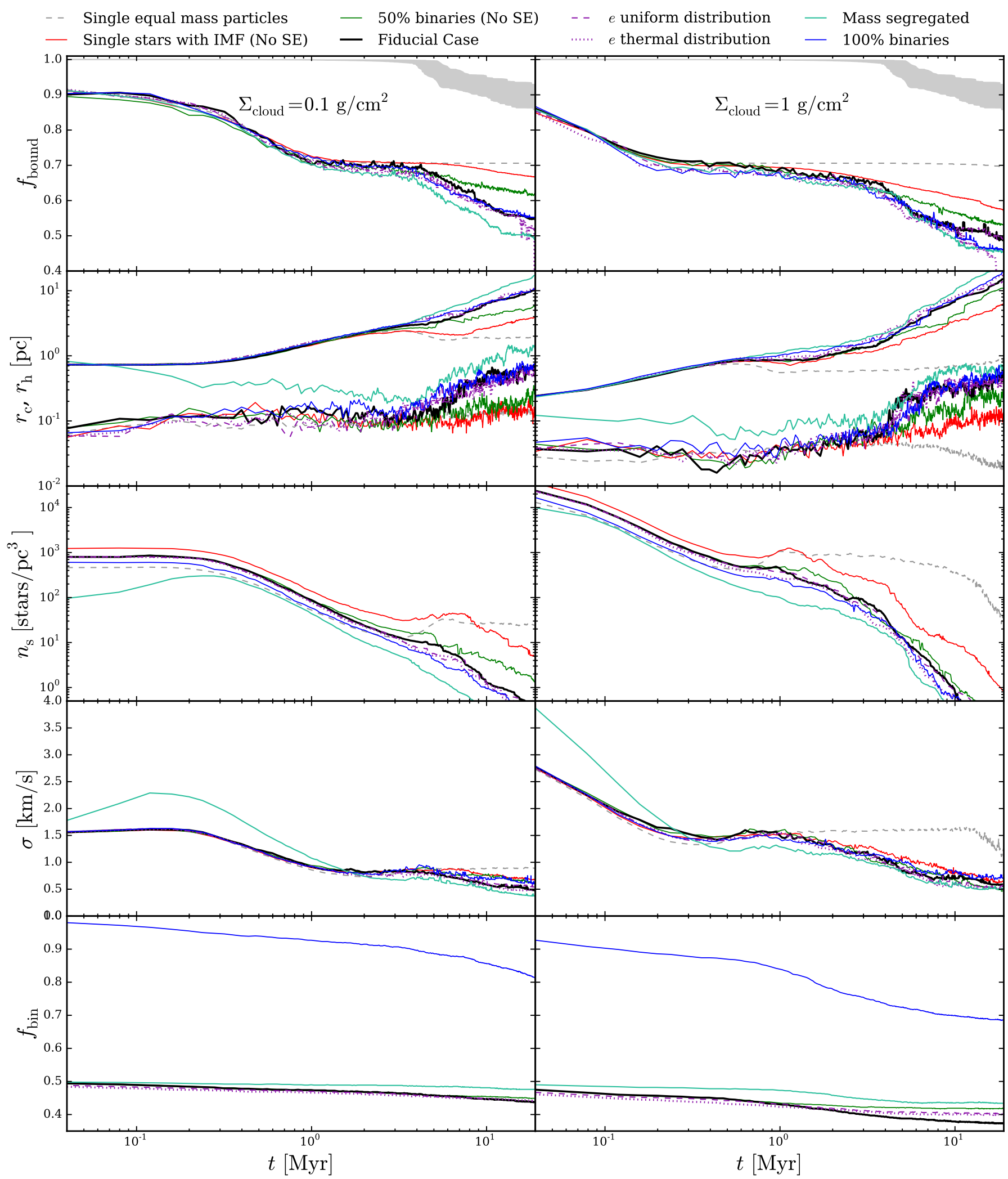

Figure 5. Time evolution of properties of fiducial clusters forming with $\mathrm{SFE}=50 \%$. Left column shows clusters forming from a $\Sigma_{\text {cloud }}=0.1$ $\mathrm{g} \mathrm{cm}^{-2}$ environment; right column from a $\Sigma_{\text {cloud }}=1 \mathrm{~g} \mathrm{~cm}^{-2}$ environment. The lines in each panel show median values calculated from the 20 simulations performed for each set. Top row shows the fraction of bound mass in the cluster relative to the initial mass, where in this figure unbound stars inside the $95 \%$ mass radius of the bound cluster are kept to show the timescale of their escape. Here we also show the fraction of total stellar mass in the fiducial simulations that remains after accounting for stellar evolution mass loss: gray shaded region shows the area between 25th and 75th percentiles. Second row shows the evolution of core radius $\left(r_{\mathrm{c}}\right)$ and half mass radius $r_{\mathrm{h}}$ for all the stars in the simulation. Third row shows the average number density of systems $\left(n_{s}\right)$, where per systems we refeer to singles and binaries, measured inside the volume defined by $r_{\mathrm{h}}$. Fourth row show the evolution of the velocity dispersion measured inside $r_{\mathrm{h}}$, and bottom row shows the evolution of the global binary fraction. 
later evolution is determined by dynamical interactions and stellar evolution.

Simulations with equal mass stars essentially do not lose further members. With an IMF, then mass segregation does lead to some additional mass loss. When including $50 \%$ primordial binaries mass loss at later times is moderately enhanced. Adding in stellar evolution, i.e., in the fiducial model, continues this trend, with a final value of $f_{\text {bound }} \simeq 0.5$. These trends are mirrored in the expansion of the clusters. Variations of binary orbital properties or primordial binary fractions are seen to have relatively minor effects.

Models with full initial mass segregation show some differences. In the case with $\Sigma_{\text {cloud }}=0.1 \mathrm{~g} \mathrm{~cm}^{-2}$, the number densities at the center are quite low initially and the core of the cluster contracts significantly. After this contraction, the number density is raised in the core, which then later expands quite rapidly. Even though number densities of these clusters are never too high, the few interactions that do occur are enough to expand the cluster and the evolution of the $50 \%$ mass radius is determined by these interactions.

In general, the star clusters presented here expand considerably regardless of the different parameters of the simulations. The amount of expansion depends on the SFE and the initial cluster density. The top panel of Figure 6 shows the ratio between the half-mass radius at the end of the simulations, $r_{\mathrm{h}, \mathrm{f}}$, and the half-mass radius at the start, $r_{\mathrm{h}, \mathrm{i}}$. The difference between the models with high and low initial densities are explained mainly by the initial velocity dispersion of the parent clump. Stars that are born unbound in the denser case escape with a higher typical velocity than the low dense case, causing the differences in the expansion. However, when considering the bound part of the cluster, the actual size of star clusters at $20 \mathrm{Myr}$ is similar regardless the initial density, as shown in the bottom panel of Figure 6. Differences between the sizes of the bound clusters arise when the SFEs are low. This is due to the fact that the crossing times of these bound systems are large $\left(t_{\mathrm{cr}} \approx 30 \mathrm{Myr}\right)$ and they have not yet achieved an equilibrium distribution by 20 Myr. Thus low SFE clusters are still in the first phase of their expansion. Regardless of the initial density, the final size of the bound systems depends mainly on the initial SFE: low SFE results in a more extended bound system.

\subsection{The effects and evolution of binaries}

Our modeling includes a full treatment of binaries, so we are able to examine their effects and evolution in detail. A binary will be significantly perturbed by an external star (or multiple) if the potential energy of the encounter is similar to that of the initial binary, i.e.,

$$
E_{\text {bin }}=-\frac{G m_{1} m_{2}}{2 a} \sim-\frac{G\left(m_{1}+m_{2}\right) m_{s}}{b},
$$

where $b$ is the closest approach of the perturber of mass $m_{s}$, and $m_{1}$ and $m_{2}$ are the primary and secondary masses of the binary, respectively. Therefore, defining $\mu \equiv m_{1} m_{2} /\left(m_{1}+m_{2}\right)$ as the reduced mass of the binary, the closest approach needed to affect binary properties is:

$$
b \sim 2 a \frac{m_{s}}{\mu} .
$$

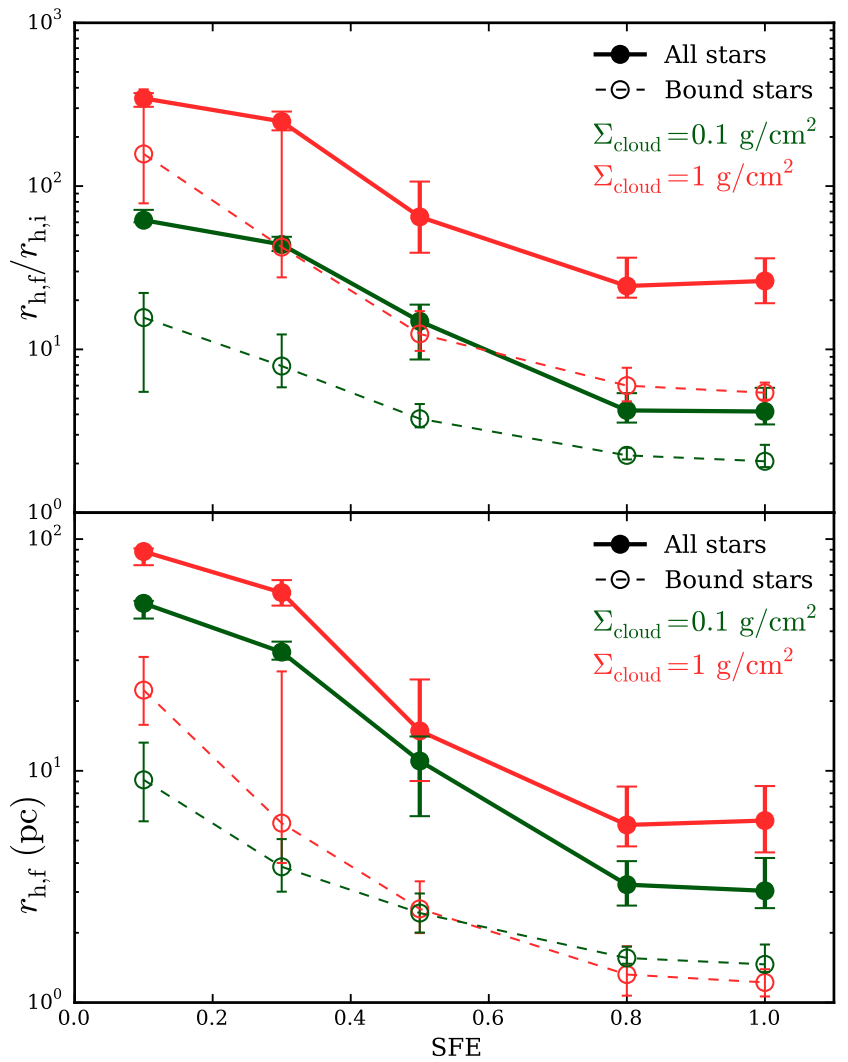

Figure 6. The size of star clusters at $20 \mathrm{Myr}$ as a function of the $\mathrm{SFE}$. Top pannel shows the final half-mass radii, $r_{\mathrm{h}, \mathrm{f}}$, compared to the initial half-mass radii, $r_{\mathrm{h}, \mathrm{i}}$. Bottom panel shows $r_{\mathrm{h}, \mathrm{f}}$ in physical units. Filled circles show measurements using all the stars; open circles using only the bound cluster. Values are medians over the 20 simulations performed for each set and errorbars shows the region between the 25 th and 75 th percentiles.

We now estimate the perturbation encounter rate of a binary of a given semimajor axis $a$ in our model star clusters. We first derive the rate assuming stars move without significant deflection, then include the effects of gravitational focusing. If we assume that the cluster has only single stars and binaries, the mean mass per system is $\left\langle m_{s}\right\rangle=\left(1+f_{\text {bin }}\right)\left\langle m_{i}\right\rangle$. If there exist higher order multiples, then $\left\langle m_{s}\right\rangle$ will be higher, however these are not included as initial conditions in our models and we will see that interactions are typically at a relatively low rate so that such multiples do not form in significant numbers during the dynamical evolution of the clusters.

The mean rate of interactions that are able to modify the properties of a binary, $\Gamma_{\mathrm{b}}$, is proportional to the cross section defined by $b$, i.e., $\pi b^{2}$, multipled by the number density of perturbing systems $n_{s}$ and a typical velocity in the cluster, i.e., the $1 \mathrm{D}$ velocity dispersion, $\sigma$. Thus the interaction rate for binaries with a given semi-major axis $a$ is

$$
\Gamma_{\mathrm{b}}=4 \pi\left(\frac{\left\langle m_{s}\right\rangle}{\mu} a\right)^{2} n_{s} \sigma .
$$

As we show in Figure 5 the effective number density in our model clusters quickly falls from initial values of $\sim 10^{3}$ to $10^{4}$ stars $/ \mathrm{pc}^{3}$ (depending on the initial environment mass surface density) to values similar to 1 stars $/ \mathrm{pc}^{3}$ at $20 \mathrm{Myr}$, in our fiducial case. The typical 

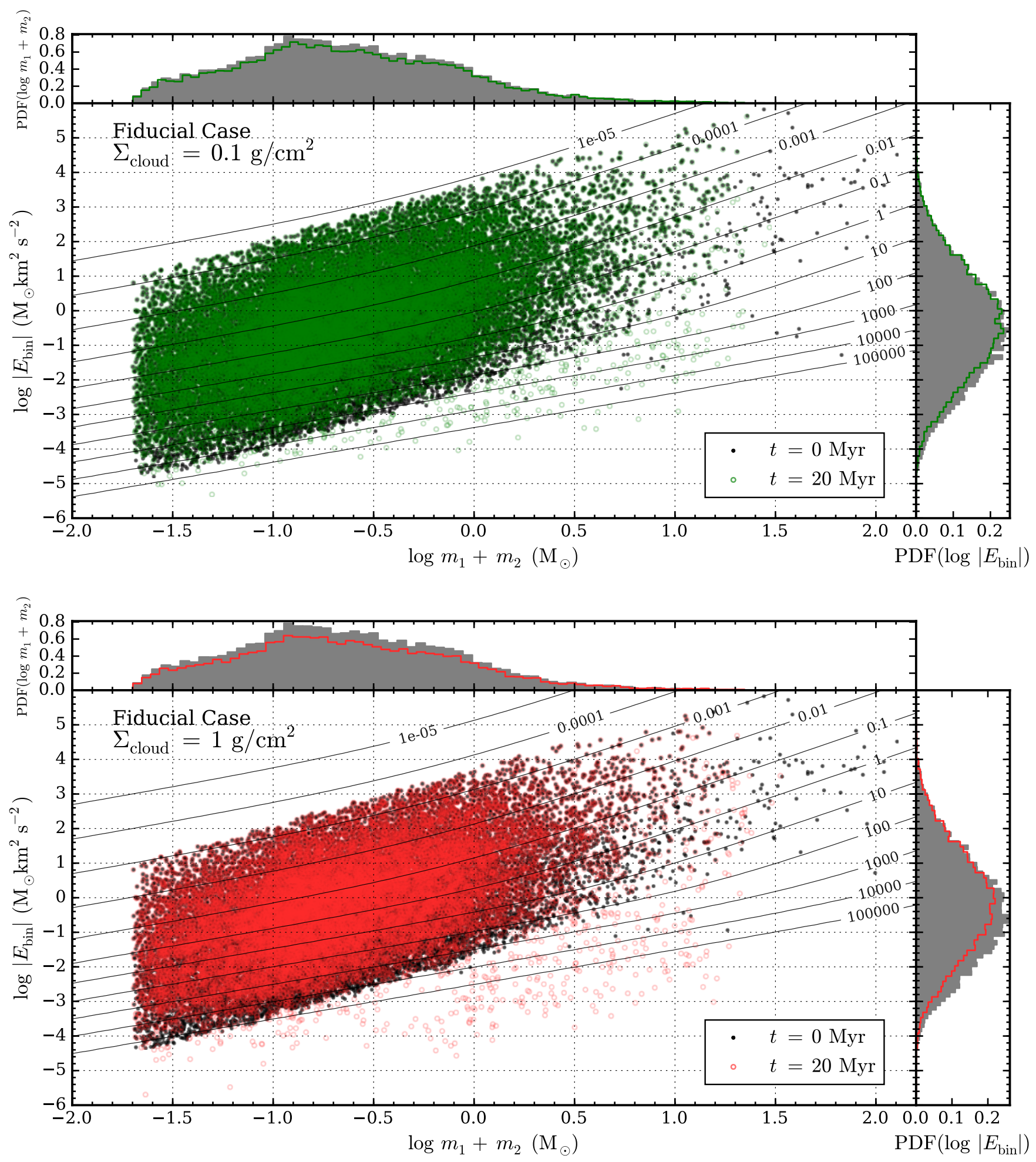

Figure 7. Binding energies as a function of total mass for primordial binaries at the start (black points) and at 20 Myr (red open circles) in the set fiducial for simulations with $\Sigma_{\text {cloud }}=0.1 \mathrm{~g} \mathrm{~cm}^{-2}$ (top panel) and $\Sigma_{\text {cloud }}=1 \mathrm{~g} \mathrm{~cm}^{-2}$ (bottom panel). Side panels show the corresponding probability distribution functions (PDFs), where the case at $20 \mathrm{Myr}$ (red lines) is normalized by the initial number of binaries. Labeled lines represent contours of $\Gamma_{\mathrm{b} \text {, eff }}$ (Eq. 22) at the beginning of the simulations in units of $\mathrm{Myr}^{-1}$, calculated using values for $n_{s}, m_{s}$ and $\sigma$ inside the half mass radius of the cluster. Few binary stars are modified in these models and typically only those with small binding energies interchange energy with the cluster. 


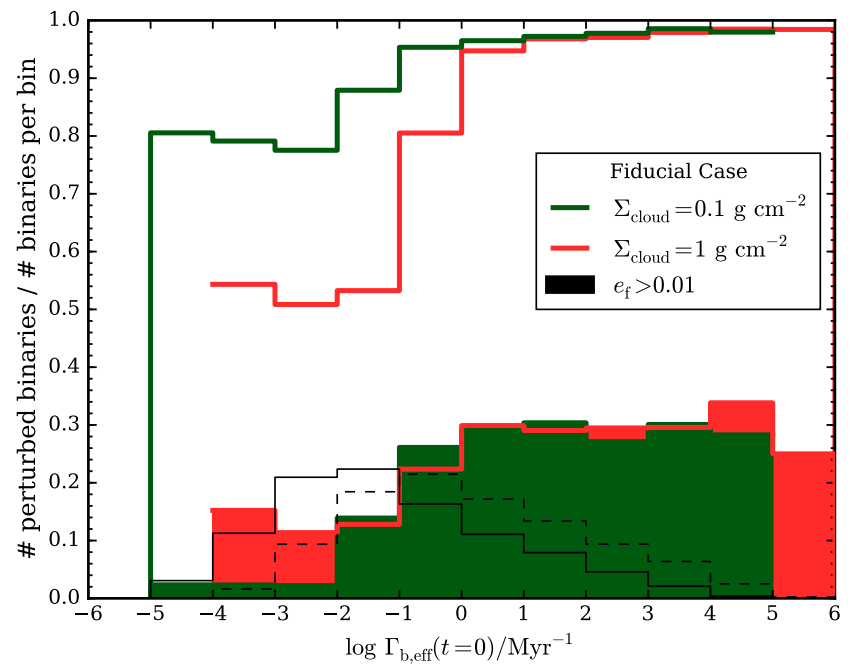

Figure 8. Number of binaries with variations in their binding energies per initial $\Gamma_{b \text {,eff }}$ bin divided by the total number of binaries on each bin for all binaries in the set fiducial, i.e. the probability of a given binary of being modified as a function of the initial $\Gamma_{\mathrm{b} \text {,eff }}$ for binaries in the set fiducial. Shaded areas represent the probability of binaries of changing their initial circular eccentricities to values $e>0.1$. Thin black lines show the total fraction of binaries in each bin for simulations with $\Sigma_{\text {cloud }}=0.1 \mathrm{~g} \mathrm{~cm}^{-2}$ (solid) and $\Sigma_{\text {cloud }}=1 \mathrm{~g} \mathrm{~cm}^{-2}$ (dashed). Only binaries with very high initial $\Gamma_{\mathrm{b} \text {,eff }}$ are able to considerably modify their initial eccentricity, however there are very small number of binaries that fulfill this condition.

velocity dispersions in the cluster, however, do not vary too much.

We can rewrite equation 18 for a given binary of semimajor axis $a$ and reduced mass $\mu$ in a more convenient way as:

$$
\begin{gathered}
\Gamma_{\mathrm{b}}(a, \mu) \lesssim 9.67 \times 10^{-3}\left(\frac{n_{s}}{10^{4} \mathrm{pc}^{-3}}\right)\left(\frac{\sigma}{2 \mathrm{~km} / \mathrm{s}}\right) \\
\times\left(\frac{a}{40 \mathrm{AU}}\right)^{2} \frac{\left\langle m_{s}\right\rangle^{2}}{\mu^{2}} \mathrm{Myr}^{-1}
\end{gathered}
$$

The above estimate does not include the effects of gravitational focusing, which will increase the effective cross section of the encounter by the factor $\mathcal{F}=\left(b_{\text {eff }} / b\right)^{2}$, where $b_{\text {eff }}$ is the effective impact parameter that leads to a closest approach $b$. Treating the binary and the perturbing system as single point masses, then conservation of energy and angular momentum and Eq. 17 imply

$$
\mathcal{F}=1+\frac{G \mu}{a \sigma^{2}}\left(\frac{m_{1}+m_{2}}{\left\langle m_{\mathrm{s}}\right\rangle}+1\right),
$$

where a more convenient way to express the last equation is:

$$
\begin{aligned}
\mathcal{F} \approx & 1+\left[5.55\left(\frac{\mu}{M_{\odot}}\right)\left(\frac{a}{40 \mathrm{AU}}\right)^{-1}\left(\frac{\sigma}{2 \mathrm{~km} / \mathrm{s}}\right)^{-2}\right. \\
& \left.\times\left(\frac{m_{1}+m_{2}}{m_{s}}+1\right)\right]
\end{aligned}
$$

Then, the effective encounter rate including gravitational focussing effects is

$$
\Gamma_{\mathrm{b}, \mathrm{eff}}=\Gamma_{\mathrm{b}} \mathcal{F}
$$

The interaction rates of binaries are mainly determined by the number density or perturbing systems, which varies by several order of magnitude over the evolution of the clusters, while none of the other environmental parameters involved in Eq. 22 vary that much. The other crucial parameters that determine the interaction rate of a binary are its own internal parameters, i.e., the internal binding energy $E_{\text {bin }}$, which determines how resistant the binary is against perturbations, and the total mass of the binary that determines the strength of the gravitational focusing effect. These parameters vary by several orders of magnitude between members in the binary population. To give a general picture of the different available interaction rates in the simulations we show the binary binding energy against binary mass in Figure 7 for binaries in the set fiducial at the start (black points) and at $20 \mathrm{Myr}$ (rad and green open circles). We show the values of $\Gamma_{b \text {,eff }}$ at the beginning of the simulation as contour lines where labels are the corresponding values in units of $\mathrm{Myr}^{-1}$.

From Figure 7 we can define the typical binary as the one having total mass of $m_{1}+m_{2} \approx 0.2 \mathrm{M}_{\odot}$ and $E_{\text {bin }} \approx 1 \mathrm{M}_{\odot} \mathrm{km}^{2} / \mathrm{s}^{2}$. Such a binary has an interaction rate of $\Gamma_{\mathrm{b} \text {,eff }} \approx 0.01 \mathrm{Myr}^{-1}$ in the low $\Sigma$ case and $\Gamma_{\mathrm{b}, \mathrm{eff}} \approx 0.2 \mathrm{Myr}^{-1}$ in the high $\Sigma$ case. These interaction rates are quite small and will fall quickly as the cluster expands. Massive binaries have higher interaction rates as they attract other systems more efficiently, however their binding energies are high and their effective impact parameters become very small. Only binaries with the smallest binding energies have high enough interaction rates to be able to interchange energy effectively with the cluster. These stars are more likely to be low mass stars.

There are several factors that determine how many interactions a binary will have during the simulations. If the binary is indeed perturbed its binding energy will change and thus also its interaction rate. The environment may vary because of several factors, e.g., the expansion of the cluster, mass segregation, binary fraction, and therefore it is quite complex to estimate the number of interactions a binary will have. However, we can use the results of our simulations and the initial $\Gamma_{\mathrm{b} \text {,eff }}$ to calculate the probability that a binary will suffer at least one important interaction during the simulation. To do so, we measure the binding energy of all binaries at $t=0$. At the end of the simulation we calculate the binding energy of the binaries that have not been disrupted and compare it with their values at the start. We define "perturbed binaries" as those with a fractional change in energy of $1 \%$.

We also measure $\Gamma_{\mathrm{b}, \text { eff }}$ according to Eq. 22 assuming density and velocity values measured inside the half mass radius of the cluster. Figure 8 shows the resulting histogram where the value of each bin has been normalized by the total amount of (undisrupted) binaries in each bin. We constructed the histograms shown in Figure 8 collecting all binaries from the 20 simulations performed for the set fiducial. We can see the correlation between the initial $\Gamma_{\mathrm{b} \text {,eff }}$ and the probability of being perturbed. There is an offset in the relation for the different initial densities. For a given initial $\Gamma_{\mathrm{b} \text {,eff }}$ the probability of suffering an encounter is higher in the low $\Sigma$ clusters. This 
seems counter-intuitive, however the rapid initial expansion of these clusters causes that the initial $\Gamma_{\mathrm{b} \text {,eff }}$ to be less representative as it does not last for long (see the number density evolution in Figure 5 for $t<0.3 \mathrm{Myr}$ ).

From all the perturbed binaries we have also highlighted the cases for which the eccentricities suffered some modification $(\Delta e>0.01)$ and displayed this probability as the shaded areas in Figure 8 . The probability of modifying the initial eccentricity appears to increase linearly with $\Gamma_{\mathrm{b} \text {,eff }}$ at first but then it remains flat for higher binary interaction rates. Even stars with high initial $\Gamma_{\mathrm{b} \text {,eff }}$ have only $\approx 30 \%$ chance of modify their initially circular orbits in a $1 \%$. We have measured only a few rare cases where the initial eccentricity increases by a significant factor.

Even though binaries with high $\Gamma_{\mathrm{b} \text {,eff }}$ have a greater chance to interact and exchange energy with the cluster, these systems are very rare, as can be seen in the thin black lines of Figure 8 that shows the total fraction of binaries in each $\Gamma_{\mathrm{b}, \text { eff }}$ bin.

The variations in these effects between our considered models is small. The models with $f_{\text {bin }}=1$ have $\Gamma_{\mathrm{b}}$ a factor 1.33 higher than simulations with $f_{\text {bin }}=0.5$, because of the effect on the number density of perturbing systems $\left(n_{s} \propto\left(1+f_{\mathrm{bin}}\right)^{-1}\right)$, and this difference becomes smaller when considering effects of gravitational focusing. Models with initial mass segregation have central number densities about 10 times lower than the fiducial case, but it increases as the cluster evolves, until a point where the few binary interactions that happen cause the cluster to expand relatively quickly. Note that most of the variables shown in Eq. 19 tend to increase towards the cluster center. Especially in the case of the mass segregated cluster, the mean mass per system is higher there, which according to Eq. 19, is one of the most important parameters since it strongly affects gravitational focusing. However, as the interactions in the centre become important the cluster expands faster, therefore is not possible to maintain high number densities.

If we assume that binaries are born as we have modeled them, i.e., with initially circular orbits, then to have significant modification of orbits, e.g., eccentricities, requires a longer high density phase, e.g., for several Myr. However, a longer timescale of cluster formation should not only keep the higher densities for longer. It also would give more time for mass segregation, which can also boost interaction rates.

\subsection{The effect of stellar evolution}

One important feature that can affect the evolution of cluster dynamics is stellar evolution, especially mass loss from winds and supernovae. Enroute to constructing our fiducial model set, we consider two sets with no stellar evolution, i.e., single_imf and binaries_50, this last one only differing from the fiducial set by having stellar evolution turned off. We also show the total mass with respect to the initial as the shaded areas in the top panels of Figure 5, with decrease by about $12 \%$ on average caused entirely by stellar evolution effects. Bound fractions at the end of the simulation in the fiducial set are $\simeq 0.55$, compared to $\simeq 0.62$ in the binaries_50 runs. Thus we see that in fact the decrease in the bound mass fraction can be explained entirely by the stellar evolution mass loss, rather than a significantly increased tendency for individual stellar members to be lost from the clusters.

Another way of losing mass from the cluster is due to the sudden ejection of the members of a binary system caused by a supernova explosion. After the supernovae explodes the binding energy suddenly drops and the system may be broken (Zwicky 1957; Blaauw 1961). The stars, both the remnant of core collapse and the secondary star of the binary, may be ejected from the cluster as runaway stars. In this case it is expected that the models with $100 \%$ binaries (binaries_100) experience a higher loss of members since all supernova occur in binary systems. However, this has only a modest effect on the bound mass fraction, as shown in Fig. 5. The orbital velocity of a $10 \mathrm{M}_{\odot}$ star in a typical binary is $\sim 0.3$ $\mathrm{km} / \mathrm{s}$ if in the peak of the period distribution, and can vary from $\sim 0.001$ to $50 \mathrm{~km} / \mathrm{s}$ if we move one $\sigma$ from the mean period. We find in our simulations that the mean escape velocity of the bound cluster varies from $\sim 7$ to $0.6 \mathrm{~km} / \mathrm{s}$ over the course of the simulation. Thus it is not certain that a binary star will be ejected from the cluster due to binary disruption. However, we have also included velocity kicks due to asymmetric SN explosions, with typical values of $\sim 100 \mathrm{~km} / \mathrm{s}$. This effect is the main factor responsible for ejections of the remnants of supernova explosions. The concomitant ejection of the secondaries will depend on the binary properties at time of supernova explosion, which for the models presented here depends mostly on primordial binary properties.

Figure 9 shows radial trajectories of the ten most massive stars in example simulations drawn from the sets of investigated models. Moving from panels (a) to (b), we see the effects of primordial binaries increasing the likelihood of dynamical ejection from an unstable multiple. Then from (b) to (c), we see the effects of stellar evolution, especially ejection of stars after supernova explosions. These types of ejections are more common than fast ejections resulting from the decay of unstable multiples. Varying the initial eccentricity distribution has only minor effects compared to the fiducial model. The fully mass-segregated case leads to the most extreme concentration of the ten most massive stars in the core of the cluster. Having $100 \%$ binaries may also lead to greater concentration of those massive star that are bound in the cluster to its core. However, note that there is a large degree of variation caused by stochastic sampling of the IMF in these examples shown in Fig. 9.

\subsection{Ejected stars and kinematic structure}

We classify the stars in three main groups: (1) unbound stars, which are born unbound from the cluster because of the initial conditions, i.e., because of the loss of gravitational potential and confining pressure due to gas expulsion; (2) bound stars, which are the stars still bound at the end of the simulation; and (3) ejected stars, which become unbound during cluster evolution. Amongst ejected stars we identify three different mechanisms that can lead to ejection: (A) "supernova ejection" either by the kick received to the core collapse remnant and/or the disruption of a binary that contained the supernova progenitor (see §3.5); (B) "dynamical ejection" due to decay of unstable triple or higher order multiple systems or due to slingshot super-elastic encounters, which leave behind a more tightly bound binary or mul- 


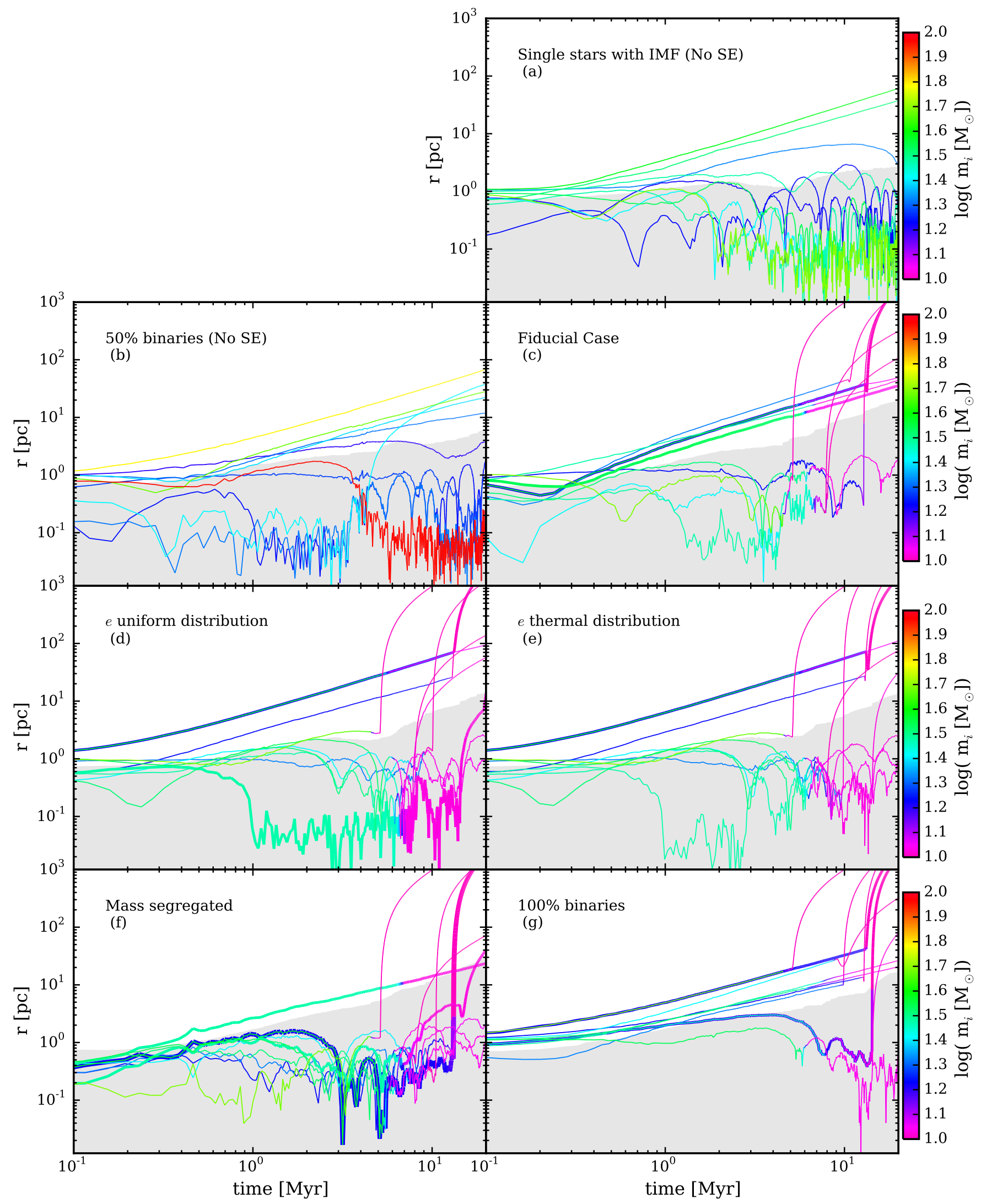

Figure 9. The evolution of the radial location of the ten most massive stars in example simulations drawn from the model sets that have an IMF and SFE of 50\%: (a) single_imf, (b) binaries_50, (c) fiducial, (d) binaries_un, (e) binaries_th (f) segregated and (g) binaries_100. Lines show stellar distances to the density center of the cluster and line colors the mass of the individual star. Each line represents a single star, and in the case of a binary there is a thinner line inside the thicker line representing the trajectory of the companion, only if the companion is also part of the 10 most massive stars, i.e., no more than 10 lines are shown on each panel. Shaded area is the region inside the half mass radius of the cluster measured with respect to the initial mass. 

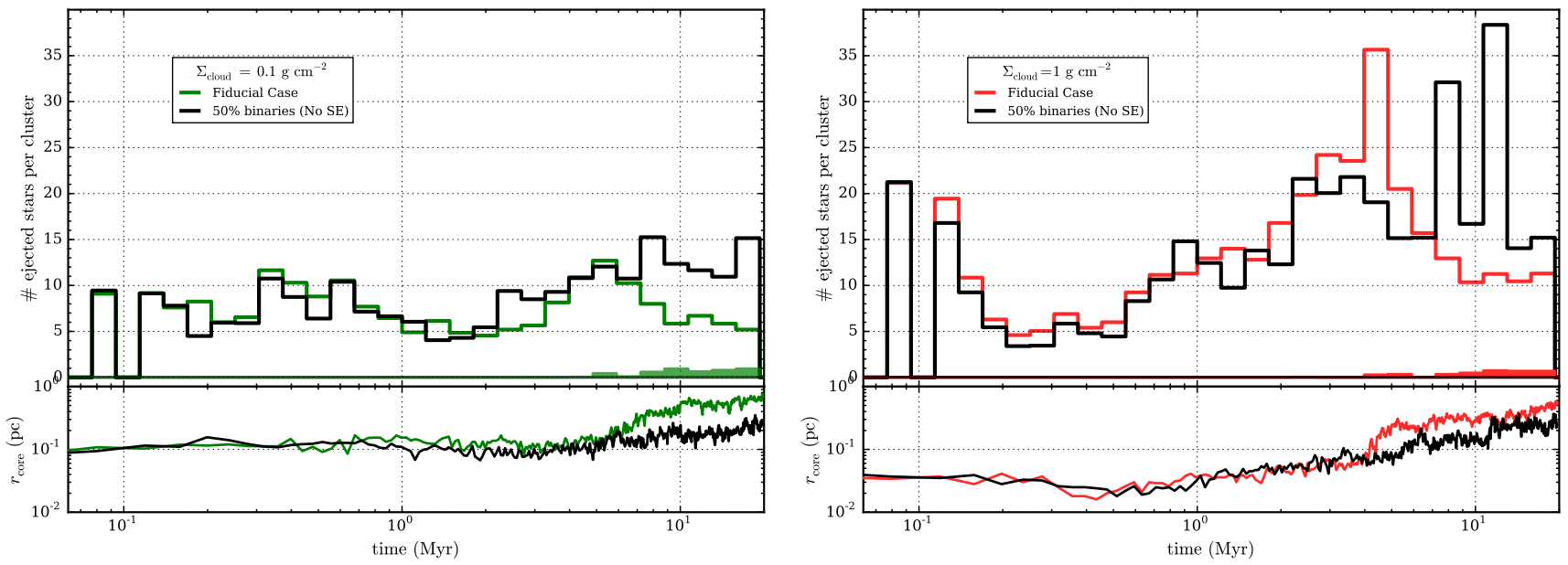

Figure 10. The number of strong dynamical ejections (see text) per logarithmic time interval per cluster simulation for the set fiducial compared with the set without stellar evolution binaries_50 for the low $\Sigma$ clump (left) and high $\Sigma$ clump (right). Shaded histograms show ejections caused by supernovae explosions. Bottom panels show the evolution of the average cluster core radii.
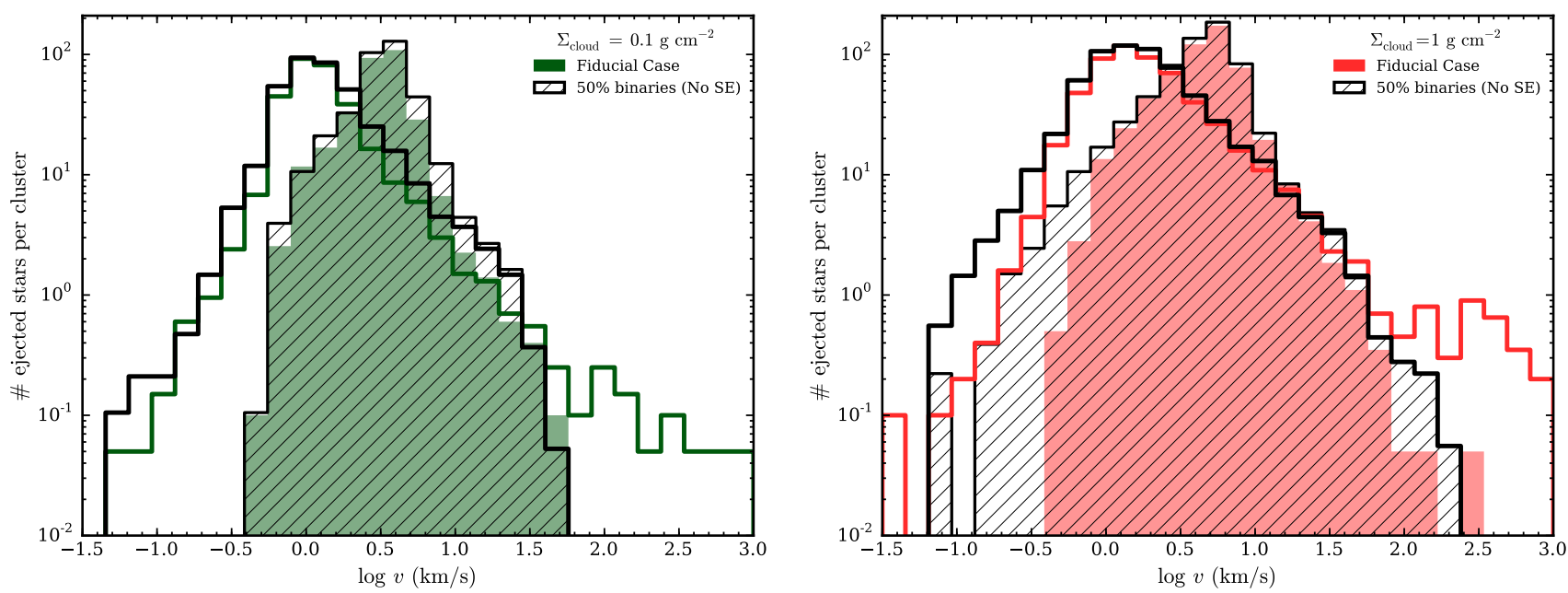

Figure 11. Velocity distribution of stars ejected dynamically in the fiducial set compared with ejected stars in the set with no stellar evolution binaries_50. Left panel show the low $\Sigma$ case and right panel the high $\Sigma$ simulations. Solid lines shows the velocity distribution at $20 \mathrm{Myr}\left(v_{\infty}\right)$ while shaded histograms shows the distribution of velocities right after ejection $\left(v_{0}\right)$. Blue dashed line represents the best fit to the set fiducial for velocities at $20 \mathrm{Myr}$, i.e. when stars already left the cluster. 
tiple; (C) "gentle ejection" where a star finds itself unbound as a result of the global evolution of the cluster potential.

In order to obtain detailed information about the ejection events we identify bound members in the simulations snapshot by snapshot, recording information about the stars the first time they appear unbound and comparing with the previous output time. We also record their positions and velocities at the end of the simulation.

We are especially interested in "strong" dynamical ejections that lead to relatively fast ejection velocities from the cluster, and identify such stars as having $\Delta T_{i} / \Delta \Omega_{i} \geq 2$ from the previous time output. Such stars will be easier to identify in proper motion studies of young clusters.

Figure 10 shows the number of such strong ejection events, including also supernova ejections, per cluster per logarithmic time interval. We compare simulations with (fiducial) and without stellar evolution (binaries_50) for the two different initial $\Sigma$ cases.

As expected, high initial $\Sigma_{\text {cloud }}$ leads to bound clusters with smaller core radii and so results in a larger rate of strong dynamical ejections than the low $\Sigma$ case. Another difference is the number of ejections after stellar evolution becomes relevant. The number of massive stars in the cluster decreases significantly after the first supernova explosions and resulting ejections. Massive stars are likely to be near the cluster center, therefore supernova explosions lead to a drop in the central density of the cluster that has a direct effect on the subsequent number of dynamical ejections, as can be seen in the anti-correlation with core radius. Cases without stellar evolution show a constant decrease in their rate of dynamical ejection events (i.e., a flat distribution in equally logarithmically spaced time bins), while in cases with stellar evolution the decrease becomes steeper after the first supernovae.

We also have information about the velocities right after ejection $\left(v_{0}\right)$ and at the end of the simulation $\left(v_{\infty}\right)$, we show the corresponding distributions in Figure 11 with shaded areas for $v_{0}$ and solid lines for $v_{\infty}$. As expected, low velocity stars show a more significant relative decrease in their velocities due to their transit out of the cluster potential. We also notice a high velocity tail of stars appearing in the fiducial case with stellar evolution: these are the result of dynamical ejection of massive stars, which later explode as supernovae resulting in a secondary kick for their remnants and any binary companions. Such a two-step ejection scenario has been proposed by Pflamm-Altenburg \& Kroupa (2010) (see also Gvaramadze et al. 2008) and has been argued to explain some O-type runaway stars and remnants with no apparent origin cluster (an alternative could be isolated massive star formation), as well as the rare observed cases of hyperfast runaways with velocities above $1000 \mathrm{~km} / \mathrm{s}$ (e.g. as in Chatterjee et al. 2005) (an alternative could be interaction with the supermassive black hole in the Galactic center).

Out of all the ejected O-type stars in our models, i.e., stars more massive than $16 \mathrm{M}_{\odot}, 35 \%$ and $22 \%$ of them were ejected dynamically in the low and high $\Sigma$ cases, respectively. While in the same order: $24 \%$ and $33 \%$ of them were ejected because of supernova explosions; and the remaining $41 \%$ and $45 \%$ were gentle ejection caused by the drop in the cluster potential or "weak" dynamical ejections (i.e., $\Delta T_{i} / \Delta \Omega_{i}<2$ ). We expect that these numbers will change when more realistic models of gradual star cluster formation are considered, since these are likely to lead to different cluster core densities.

Figure 12 shows the normalized velocity versus mass (left panels) and normalized velocity versus radial distance from cluster center (right panels) for all the stars in the simulations of the fiducial set (see Figure 15 in the Appendix A for same plots for other sets) with low (top panels) and high (bottom panels) values of $\Sigma_{\text {cloud }}$. Velocities are normalized by the mass average velocity dispersion of the parent clump (see $\sigma$ in Table 2 and Eq. 9). Masses show the final mass of the single stars (filled circles) or combined binary (filled stars).

With exception of massive stars undergoing supernova explosions, unbound stars (green symbols) do not change their velocities significantly on leaving the cluster. They have a peak on the PDF at $\sigma$, below the initial mean escape velocity of the cluster, $v_{\text {esc,i }}$, shown by a green dashed line in Figure 12. Figure 15 shows that the width of the velocity PDF of unbound stars is quite constant over all the different simulation sets.

Ejected stars have a velocity PDF that peaks between those of the unbound and bound stars. This is because the escape velocity decreases with time as the cluster expands and loses members. As discussed earlier, a cluster born in a denser state expands more quickly and at 20 Myr its half mass radius is about 10 times larger than the same cluster born in the lower density state. This leads to a larger relative decrease in the mean escape speed of the cluster over the $20 \mathrm{Myr}$ of evolution followed by these simulations, compared to the low $\Sigma$ case. This in turn causes a broadening of the ejected star velocity PDF. Otherwise, the widths of the velocity PDFs do not vary much between simulations sets.

Bound and unbound stars are more clearly distinguished in the velocity versus radial distance diagram. Bound stars have, in general, higher velocities at the center of the cluster and lower velocities at the outskirts. They thus populate different areas from the unbound and ejected stars: higher velocities of these stars carry them further from the cluster, modulated also by the time when they were ejected. Supernova induced velocity kicks also lead to modification of a small fraction of stars in this diagram. The group of neutron stars seen in the velocity-mass diagram is another manifestation of such effects. Their velocities are a direct result of the assumed Maxwellian distribution for supernova-induced kick velocities with $\sigma=265 \mathrm{~km} \mathrm{~s}^{-1}$.

Figure 13 shows the IMF of the three different classes of stars: bound, unbound and ejected. The IMF of the unbound group (green histogram) mirrors the assumed primordial distribution; stars from all masses are initially randomly distributed and are equally likely to be born unbound. The initially bound cluster shows the same pattern, however as the cluster evolves and stars are ejected, almost all massive stars are lost resulting in the black histogram shown in Figure 13. Thus the IMF of the ejected group (red histogram) shows a clear signature of being top heavy - mostly a consequence of stellar evolution. Eventually, a large majority of the stars able 

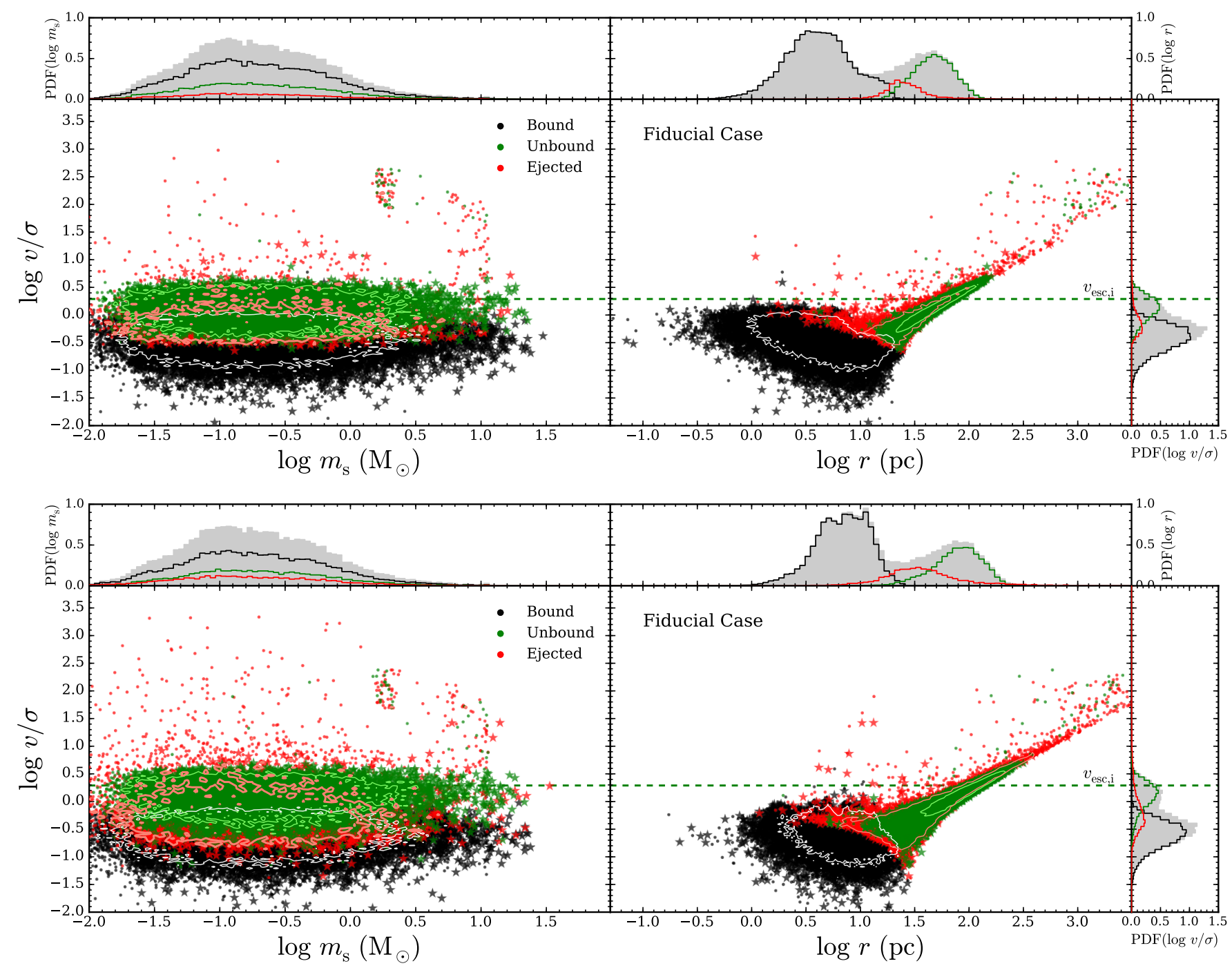

Figure 12. Normalized velocity versus mass (left panels) and versus distance (right panels) for all stars in the fiducial case for simulations with $\Sigma_{\text {cloud }}=0.1 \mathrm{~g} \mathrm{~cm}^{-2}$ (top) and $\Sigma_{\text {cloud }}=1 \mathrm{~g} \mathrm{~cm}^{-2}$ (bottom). Stars are separated in three groups: bound stars (black); stars born unbound (green); and ejected stars (red). Different symbols represent either if the star is a single (circle) or a binary (star). Velocity values are normalized by the mass average velocity dispersion of the parent clump (see 2). Small top and side panels show the PDFs considering all stars in the set (gray shaded area), and the fraction of the PDFs that correspond to each group of stars (lines). The escape velocity from the stellar cluster at its surface at the start of the simulation is shown by a green dashed line. In order to show some of the structure hiding in the cloud of points, contours that contains $90 \%$ of the stars on each set are shown in a lighter color, i.e., white for bound, light green for unbound, and light red for ejected stars.

to explode as supernovae are ejected from the cluster. When comparing with other simulation sets (see Figure 17), we see that even before including stellar evolution the IMF of ejected stars is already top heavy, which is a result only of dynamical ejections. However, this effect is not strong enough to significantly change the shape of the bound cluster IMF.

Further variations from the set fiducial do not change these results significantly, with exception of the segregated sets. Initial extreme mass segregation causes a very different evolution in the three groups of stars. Stars born unbound are preferentially the lowest mass stars, therefore the initially bound clusters have top heavy IMFs. Later evolution causes the cluster to lose its massive stars. However, such extreme mass segregation is a very idealized model that is not expected to be a very realistic description of observed clusters.

\subsection{Radial structure}

We now summarize the evolution of various radial distributions of stellar properties in the clusters, which, in their projected forms, are one of the most direct observables of real systems (see Figure 14 for results for the fiducial set of simulations and Appendix A for the other sets). For the low and high $\Sigma_{\text {cloud }}$ cases we show radial profiles for volume density (panels a and b), projected mass surface density (panels $\mathrm{c}$ and $\mathrm{d}$ ), projected $1 \mathrm{D}$ velocity dispersion $\sigma_{1 \mathrm{D}}$ (panels e and $\mathrm{f}$ ) and projected mean stellar mass $\left\langle m_{\mathrm{i}}\right\rangle$ (panels $\mathrm{g}$ and $\mathrm{h}$ ).

These figures show the expansion of the clusters and the flattening of the initial power law density profile, i.e., development of a constant density core. Stars born unbound separate from the cluster as it evolves and appear as an excess "halo" around the bound cluster.

As expected, the velocity dispersion profiles of the 


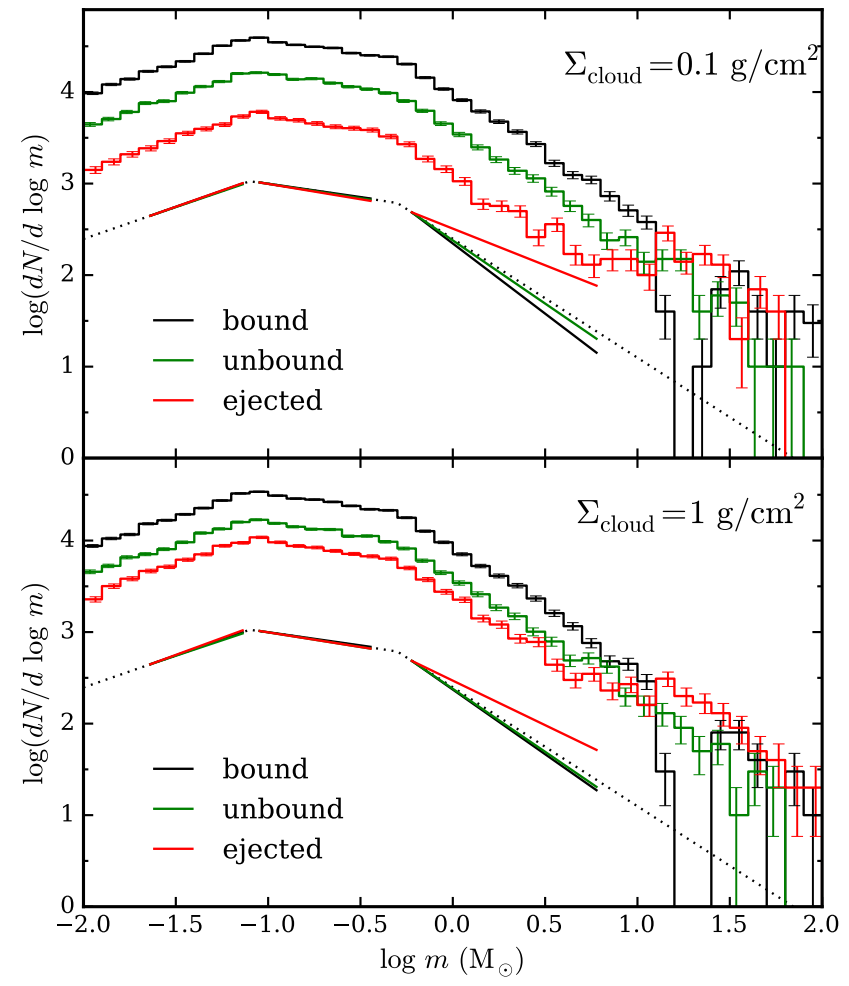

Figure 13. Collected IMFs for all the stars in the fiducial case separated in bound (black steps), unbound (green steps) and ejected (red steps) stars as in Figure 12. Top panel shows simulations with $\Sigma_{\text {cloud }}=0.1 \mathrm{~g} / \mathrm{cm}^{2}$ and bottom panel simulations with $\Sigma_{\text {cloud }}=1 \mathrm{~g} / \mathrm{cm}^{2}$. Lines below the steps shows the shape of the best fit on each range of masses for each group of stars over-plotted on the canonical IMF used as initial condition for comparison. In all groups the IMF below $0.5 \mathrm{M}_{\odot}$ is the same and do not change from the original, but the group of ejected stars shows a top heavy IMF.

bound stars show a general trend of evolving towards smaller values as the clusters expand. This can also be seen in Figure 5 with a similar evolution for all the different simulation sets. The velocity profiles are relatively flat, but with a modest tendency to decrease in the outer regions.

The last row of Figure 14 shows the average stellar mass per system, i.e., masses of binary stars are combined. By this metric, we do not see significant signatures of mass segregation developing in the clusters. Then at later times, stellar evolution, i.e., wind mass loss and supernovae, acts to remove massive stars. Stochastic effects due to IMF sampling are still noticeable, even when averaging over 20 clusters.

The most extreme primordially segregated case we considered (set segregated) is able to maintain its mass segregation for much of the 20 Myr evolution (note, stars born unbound are mostly low mass stars), but it becomes less prominent in the high $\Sigma$ case since massive stars are ejected more efficiently by dynamical interactions. Then after $3 \mathrm{Myr}$, massive star start to be lost due to stellar evolution (supernova) effects.

\section{DISCUSSION AND CONCLUSIONS}

We have presented a first modeling of the dynamical evolution of star clusters forming with initial conditions prescribed by the Turbulent Clump Model of McKee \& Tan (2003). These initial conditions involve idealized de- scriptions of star-forming protocluster clumps as singular polytropic spheres in virial equilibrium (including effects of large scale magnetic field support) and pressure equilibrium with a surrounding cloud medium (i.e., clump radius is set by truncation of the polytropic sphere where local clump pressure matches ambient cloud pressure, with the latter assumed to be due to the self-gravitating weight of the larger-scale cloud of given mass surface density, $\left.\Sigma_{\text {cloud }}\right)$. In this first paper we have assumed, for simplicity, that star clusters are formed instantaneously with a spatially uniform star formation efficiency. Subsequent papers will build realism to this model, in particular allowing for the effects of gradual star formation.

The first consequence of the above assumptions is that stars follow the same spatial and kinematic distributions of the parent clump, which sets the main difference between this and previous related studies in which velocity profiles of the star clusters are constructed using either isotropic velocity distributions, using methods described by Aarseth et al. (1974) (e.g., Goodwin \& Bastian 2006) or assuming the stars are in equilibrium with their natal gas at the onset of gas expulsion (e.g., Baumgardt \& Kroupa 2007). A system in equilibrium has a velocity dispersion profile that decreases with radius. The Turbulent Clump Model, however, involves larger velocity dispersions at larger scales, so stars born on the outskirts of the clump are less likely to remain bound to the cluster. Conversely, these models contain a central, relatively tightly bound central region.

One crucial parameter that determines the amount of retained mass after gas is expelled in this model is the contribution of magnetic fields to the support of the parent clump. Without magnetic field support the virial ratios at the onset of gas expulsion are relatively high even for SFE of $100 \%$. We can infer from our results that without magnetic fields $100 \%$ SFE would result in clusters that retain only $\sim 40 \%$ of the stars (see Figures 1 and 2). However, the models presented here are the worst case scenario in terms of cluster survivability. It has been shown that gradual gas expulsion increases the amount of stellar mass retained (e.g., Mathieu 1983; Baumgardt \& Kroupa 2007; Smith et al. 2013a). Another factor is the central star to gas mass ratio at the onset of gas expulsion (e.g., Kruijssen et al. 2012). Our models have assumed a spatially uniform SFE, but the local SFE may be raised either by dynamical cluster relaxation before gas expulsion (Smith et al. 2011; Farias et al. 2015) or by the star formation process itself, which has been argued to be faster and globally more efficient in the densest regions of the clump (Kruijssen et al. 2012; Kruijssen 2012; Parmentier \& Pfalzner 2013).

The fiducial clusters that we have simulated show rapid expansion, even just considering their bound members. For example, the half-mass radii show dramatic (factors of several) expansion after $1 \mathrm{Myr}$, especially in the high $\Sigma_{\text {cloud }}=1 \mathrm{~g} \mathrm{~cm}^{-2}$ case. Thus for these models to explain observed young star clusters of a given age, mass and mass surface density (see, e.g., Fig. 1 of Tan et al. 2014), would require initial clumps that have mass surface densities at least ten times greater. There is limited evidence for such dense starless clumps (Tan et al. 2014; see also Walker et al. 2016). This may indicate that some aspect of the model needs to be modified, such as 
Fiducial Case

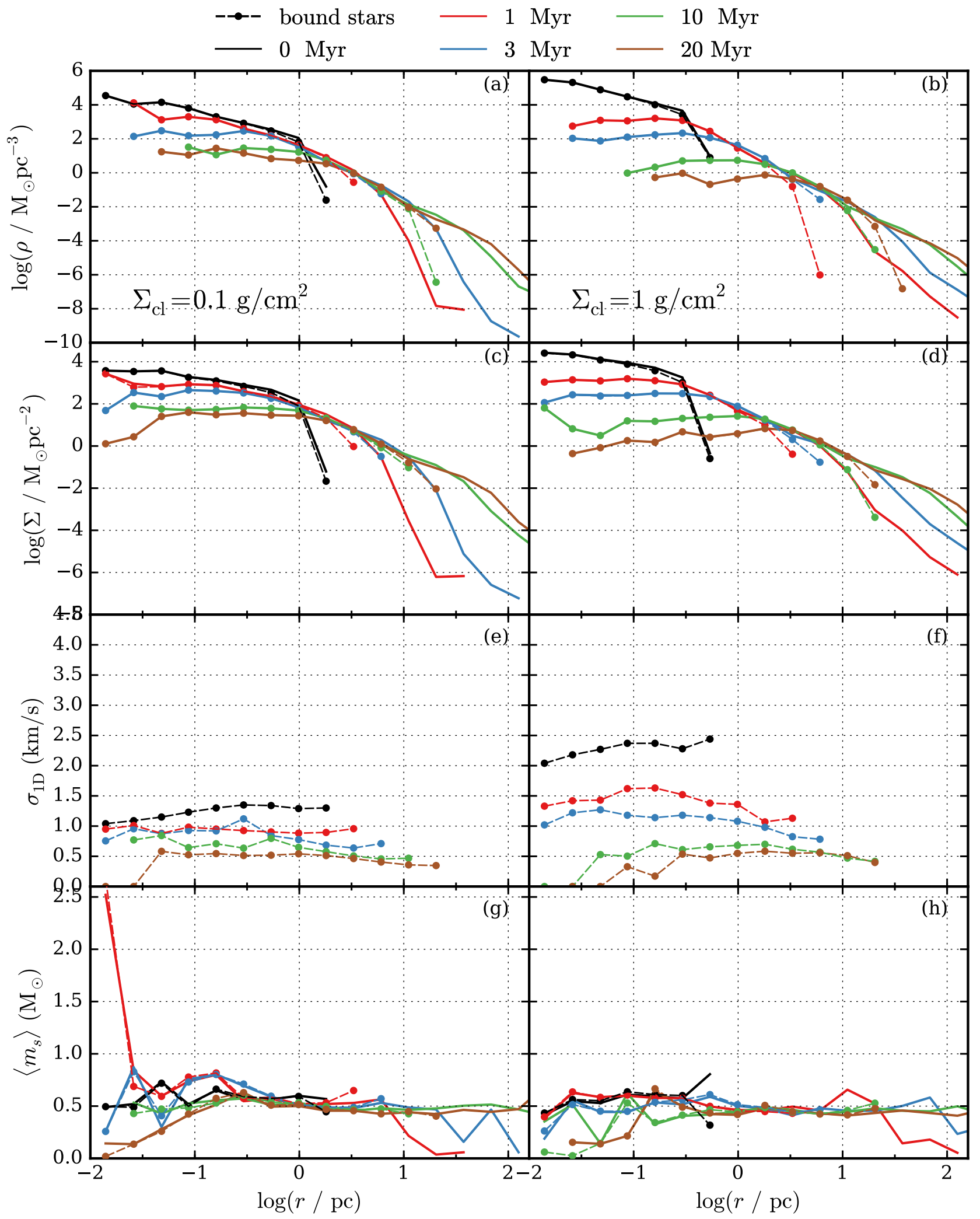

Figure 14. Radial profiles of the fiducial simulations at: 0 Myr (black); 1 Myr (red); 3 Myr (blue); 10 Myr (green); 20 Myr (brown), with results for low $\Sigma_{\text {cloud }}$ shown in the left column and high $\Sigma_{\text {cloud }}$ in the right column. Dashed lines and circles show the mean values of the 20 realizations for the bound clusters, while solid lines show these averages for the total stellar population. Top row, panels (a) and (b): stellar volume density radial profile as a function of spherical radial coordinate from cluster center. 2nd row, panels (c) and (d): mass surface density profile as a function of projected radial coordinated from cluster center. 3rd row, panels $(e)$ and $(f)$ : 1D velocity dispersion profiles as a function of projected radius. 4th row, panels $(g)$ and $(h)$ : average mass per system profile as a function of projected radius. Note, in the 3rd row $\sigma_{1 \mathrm{D}}$ for all stars has been omitted because of the large variations caused by runaway stars. Note also, binary stars are treated as unresolved systems, i.e., we use their combined mass, position and velocity to construct each profile. 
the assumption of instantaneous star formation and gas expulsion.

Another feature of our work has been the full treatment of binaries, given assumed primordial binary properties. The processing of these binaries during the early phases of the dynamical evolution of star clusters can be a diagnostic of the process, i.e., by comparing their properties with observed field star and embedded cluster binary properties. We have also seen that binaries affect some aspects of the dynamical evolution of the cluster, in particular by enhancing the rate of dynamical ejection of stars. We consider that our main results on this topic so far are to show the relative importance of binaries in our model clusters depending on their input assumptions. In the clusters that we have investigated in this paper there has been relatively little processing of the average initial binary properties given the fairly rapid expansion of the clusters from their initial dynamical states. These results provide a baseline for comparison of future models of gradual star cluster formation.

Since we have a full treatment of binaries and stellar evolution, we are also able to make predictions for the properties of ejected stars, including via dynamical ejection from unstable triples and higher order multiples and from supernova explosions. The models presented here are only able to reproduce high velocity runaway stars by supernovae kicks. Only the densest initial conditions lead to dynamical ejection of stars at speeds $>100 \mathrm{~km} \mathrm{~s}^{-1}$ (but less than one per cluster) and we have not obtained dynamical ejection of any massive stars at speeds $>20 \mathrm{~km} \mathrm{~s}^{-1}$. This probably again indicates a need for more gradual models of star formation that retain a dense cluster core for a larger number of crossing times.

In the context of our presented models that apply in the limit of fast, i.e., "instantaneous," star cluster formation, our main conclusions are:

1. Magnetic fields that partially support the parent clump against collapse are a key factor that determines the initial velocity and bound mass of the new born cluster. Star clusters born from clumps with no magnetic field support would need very high $\mathrm{SFE}(>80 \%)$ to retain a significant part of the stellar mass after gas is expelled.

2. Regardless of the different initial surface densities, the mass of the bound cluster that emerges from its natal gas is determined by the SFE. However, the expansion rate of the unbound stellar population is determined by the typical internal velocity of the parent clump. This means that clusters born in high mass surface density environments will produce unbound populations that expand more rapidly than those produced from clusters formed in low mass surface density environments.

3. The rapid early expansion of the bound clusters imply that at least $\sim 10 \times$ higher mass surface density starless clumps are needed to explain observed young $(\sim 1$ Myr old) clusters that have stellar mass surface densities $\gtrsim 0.1 \mathrm{~g} \mathrm{~cm}^{-2}$, but there is limited evidence for such clumps. This may indicate a need for more gradual models of star cluster formation.
4. The low interaction rates of binaries in our simulated clusters lead to only minor modifications of average initial binary properties. If star cluster formation is rapid, then the present day observed binary properties have not changed much from their primordial distributions, except for those effects induced by stellar evolution.

5. Based on velocity-position diagrams, it is possible to distinguish the bound cluster from the unbound stars and we expect that this is made easier if gas is expelled quickly. Such diagrams also give us information on the initial properties of the parent clump, such as the initial escape velocity from the cluster.

6. High velocity runaway stars in the clusters simulated so far are mostly due to supernova explosions that disrupt binaries. Such statistics likely indicate, again, that star cluster formation does not proceed in such a rapid manner.

It remains to be determined how the above metrics will vary for models of star clusters forming from turbulent clumps that involve gradual formation of stars and gradual gas expulsion. In addition, correlated spatial and kinematic substructures associated with turbulence and potential infalling accretion flows need to be incorporated into these models. Variations in global geometry, e.g., elongation of the clump into more filamentary configurations, also need to be explored. A wider range of initial conditions of clump properties and assumed star formation efficiencies, primordial mass segregation and primordial binary properties need to be investigated for this model family. By an eventual comparison of model results with observed kinematic properties of young clusters, we hope to constrain star and star cluster formation theories.

We thank Nicola Da Rio and Antonio Ordoñez for helpful discussions. We acknowledge support from Hubble Space Telescope Cycle 23 Theory Grant 14317, The Orion Experiment (PI: Tan).

\section{REFERENCES}

Aarseth, S. J. 2003, Gravitational N-Body Simulations, 430 Aarseth, S. J., \& Heggie, D. C. 1998, MNRAS, 297, 794

Aarseth, S. J., Henon, M., \& Wielen, R. 1974, A\&A, 37, 183

Banerjee, S., \& Kroupa, P. 2015, ArXiv e-prints, arXiv:1512.03074

Basri, G., \& Reiners, A. 2006, AJ, 132, 663

Bastian, N., \& Goodwin, S. P. 2006, MNRAS, 369, L9

Baumgardt, H., \& Kroupa, P. 2007, MNRAS, 380, 1589

Blaauw, A. 1961, Bull. Astron. Inst. Netherlands, 15, 265

Boily, C. M., \& Kroupa, P. 2003, MNRAS, 338, 673

Brinkmann, N., Banerjee, S., Motwani, B., \& Kroupa, P. 2016, ArXiv e-prints, arXiv:1611.05871

Butler, M. J., \& Tan, J. C. 2009, ApJ, 696, 484

- 2012, ApJ, 754, 5

Chatterjee, S., \& Tan, J. C. 2012, ApJ, 754, 152

Chatterjee, S., Vlemmings, W. H. T., Brisken, W. F., et al. 2005, ApJL, 630, L61

Chen, H.-C., \& Ko, C.-M. 2009, ApJ, 698, 1659

Close, L. M., Siegler, N., Freed, M., \& Biller, B. 2003, ApJ, 587, 407

Dale, J. E., Ercolano, B., \& Bonnell, I. A. 2015, MNRAS, 451, 987

Duquennoy, A., \& Mayor, M. 1991, A\&A, 248, 485 
Elmegreen, B. G. 2000, ApJ, 530, 277

-. 2007, ApJ, 668, 1064

Farias, J. P., Smith, R., Fellhauer, M., et al. 2015, MNRAS, 450, 2451

Fischer, D. A., \& Marcy, G. W. 1992, ApJ, 396, 178

Geyer, M. P., \& Burkert, A. 2001, MNRAS, 323, 988

Ginsburg, A., Bressert, E., Bally, J., \& Battersby, C. 2012, ApJL, 758, L29

Goodwin, S. P., \& Bastian, N. 2006, MNRAS, 373, 752

Gutermuth, R. A., Megeath, S. T., Myers, P. C., et al. 2009, ApJS, 184, 18

Gvaramadze, V. V., Gualandris, A., \& Portegies Zwart, S. 2008, MNRAS, 385, 929

Hartmann, L., \& Burkert, A. 2007, ApJ, 654, 988

Heggie, D. C. 1975, MNRAS, 173, 729

Hills, J. G. 1975, AJ, 80, 809

Hobbs, G., Lorimer, D. R., Lyne, A. G., \& Kramer, M. 2005, MNRAS, 360, 974

Hurley, J. R., Pols, O. R., \& Tout, C. A. 2000, MNRAS, 315, 543

Hurley, J. R., Tout, C. A., \& Pols, O. R. 2002, MNRAS, 329, 897

Kaczmarek, T., Olczak, C., \& Pfalzner, S. 2011, A\&A, 528, A144

Kouwenhoven, M. B. N., Goodwin, S. P., Parker, R. J., et al. 2010, MNRAS, 404, 1835

Kroupa, P. 2001, MNRAS, 322, 231

Kroupa, P., \& Burkert, A. 2001, ApJ, 555, 945

Kroupa, P., Petr, M. G., \& McCaughrean, M. J. 1999, New A, 4, 495

Kruijssen, J. M. D. 2012, MNRAS, 426, 3008

Kruijssen, J. M. D., Maschberger, T., Moeckel, N., et al. 2012 , MNRAS, 419, 841

Lada, C. J., Margulis, M., \& Dearborn, D. 1984, ApJ, 285, 141

Ma, B., Tan, J. C., \& Barnes, P. J. 2013, ApJ, 779, 79

Mason, B. D., Gies, D. R., Hartkopf, W. I., et al. 1998, AJ, 115, 821

Mathieu, R. D. 1983, ApJL, 267, L97

McKee, C. F., \& Ostriker, E. C. 2007, ARA\&A, 45, 565

McKee, C. F., \& Tan, J. C. 2003, ApJ, 585, 850

Myers, A. T., Klein, R. I., Krumholz, M. R., \& McKee, C. F. 2014, MNRAS, 439, 3420

Nakamura, F., \& Li, Z.-Y. 2007, ApJ, 662, 395

-. 2014, ApJ, 783, 115

Padoan, P., Haugbølle, T., \& Nordlund, Å. 2012, ApJL, 759, L27

-. 2014, ApJ, 797, 32
Parker, R. J., Goodwin, S. P., \& Allison, R. J. 2011, MNRAS, 418, 2565

Parker, R. J., Goodwin, S. P., Kroupa, P., \& Kouwenhoven,

M. B. N. 2009, MNRAS, 397, 1577

Parker, R. J., \& Meyer, M. R. 2014, MNRAS, 442, 3722

Parker, R. J., Wright, N. J., Goodwin, S. P., \& Meyer, M. R. 2014, MNRAS, 438, 620

Parmentier, G., \& Pfalzner, S. 2013, A\&A, 549, A132

Pfalzner, S., \& Kaczmarek, T. 2013, A\&A, 555, A135

Pfalzner, S., Vincke, K., \& Xiang, M. 2015, A\&A, 576, A28

Pflamm-Altenburg, J., \& Kroupa, P. 2010, MNRAS, 404, 1564

Preibisch, T., Balega, Y., Hofmann, K.-H., Weigelt, G., \& Zinnecker, H. 1999, New A, 4, 531

Price, D. J., \& Bate, M. R. 2009, MNRAS, 398, 33

Proszkow, E.-M., \& Adams, F. C. 2009, ApJS, 185, 486

Raghavan, D., McAlister, H. A., Henry, T. J., et al. 2010, ApJS, 190, 1

Rathborne, J. M., Jackson, J. M., \& Simon, R. 2006, ApJ, 641, 389

Reggiani, M. M., \& Meyer, M. R. 2011, ApJ, 738, 60

Schuller, F., Menten, K. M., Contreras, Y., et al. 2009, A\&A, 504, 415

Smith, R., Fellhauer, M., Goodwin, S., \& Assmann, P. 2011, MNRAS, 414, 3036

Smith, R., Goodwin, S., Fellhauer, M., \& Assmann, P. 2013a, MNRAS, 428, 1303

Smith, R., Sánchez-Janssen, R., Fellhauer, M., et al. 2013b, MNRAS, 429, 1066

Tan, J. C., Beltrán, M. T., Caselli, P., et al. 2014, Protostars and Planets VI, 149

Tan, J. C., Kong, S., Butler, M. J., Caselli, P., \& Fontani, F. 2013, ApJ, 779, 96

Tan, J. C., Krumholz, M. R., \& McKee, C. F. 2006, ApJL, 641, L121

Tutukov, A. V. 1978, A\&A, 70, 57

Walker, D. L., Longmore, S. N., Bastian, N., et al. 2016, MNRAS, 457,4536

Wang, L., Spurzem, R., Aarseth, S., et al. 2015, MNRAS, 450, 4070

Zwicky, F. 1957, Morphological astronomy

APPENDIX

ANCILLARY RESULTS FOR THE FULL SET OF SIMULATIONS 

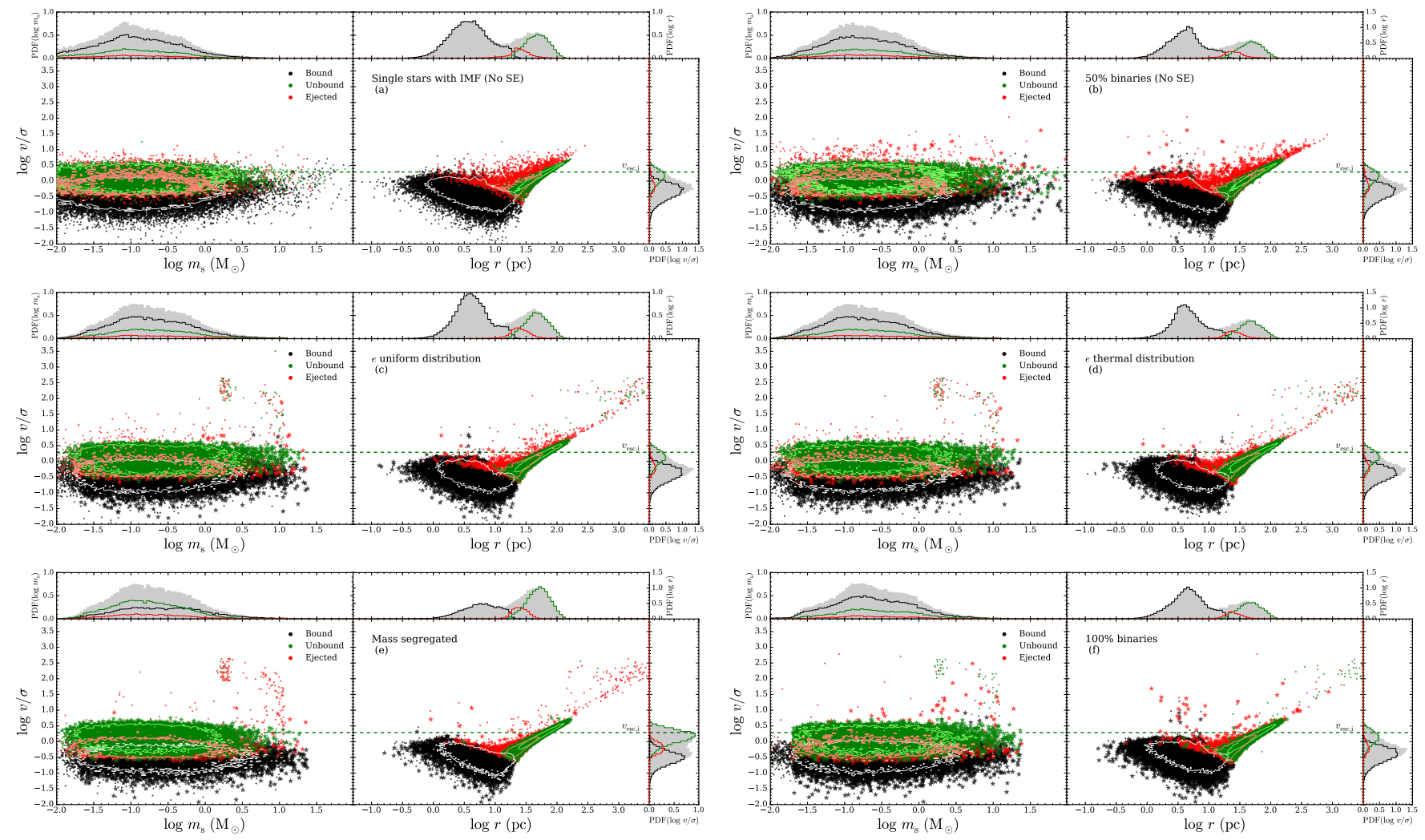

Figure 15. Same as Figure 12, but now for simulations sets: (a) single_imf, (b) binaries_50, (c) binaries_un, (d) binaries_th, (e) segregated and (f) binaries_100, all with $\Sigma_{\text {cloud }}=0.1 \mathrm{~g} / \mathrm{cm}^{2}$.
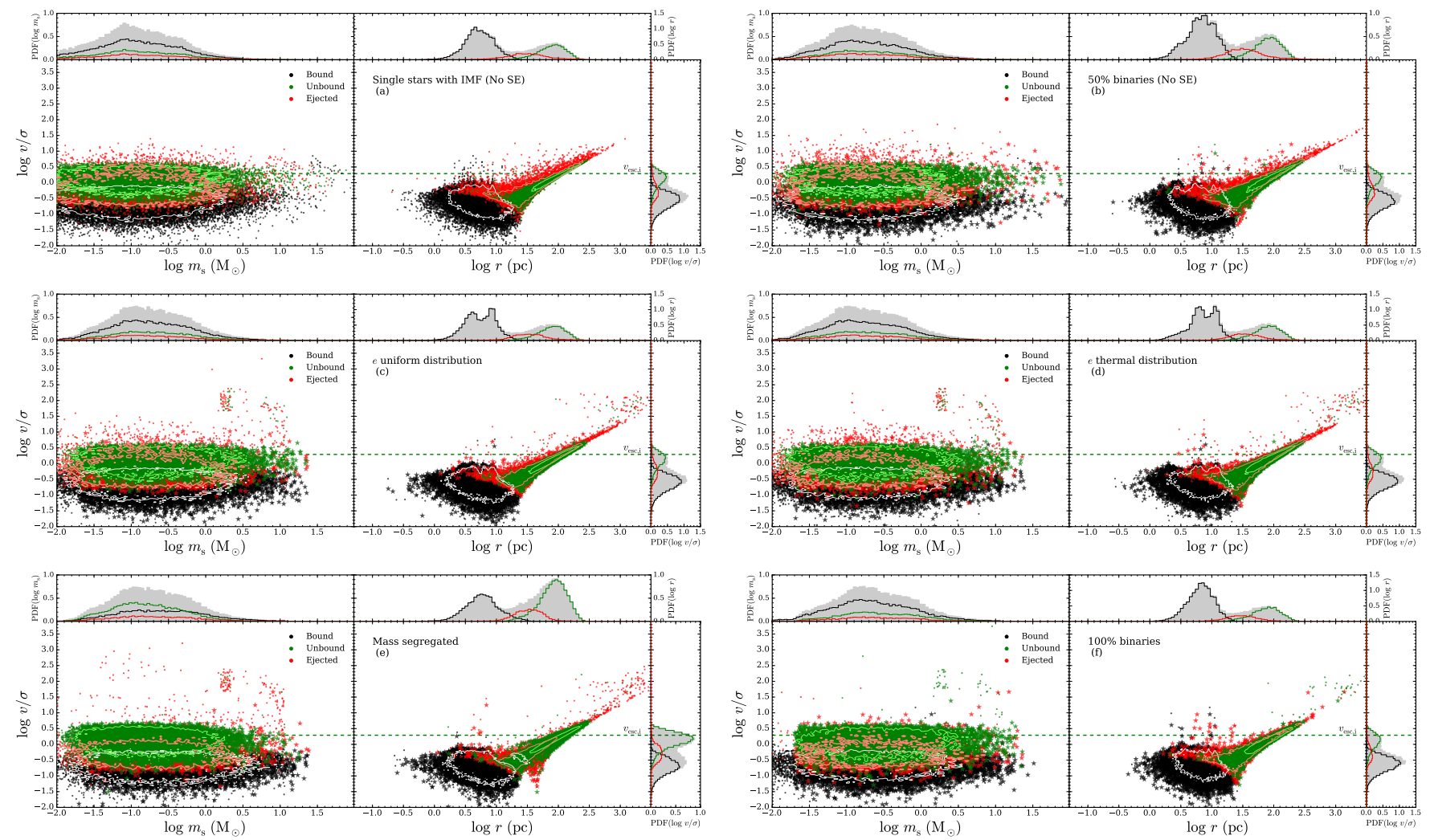

Figure 16. Same as Figure 15, but now for simulations with $\Sigma_{\text {cloud }}=1 \mathrm{~g} / \mathrm{cm}^{2}$. 

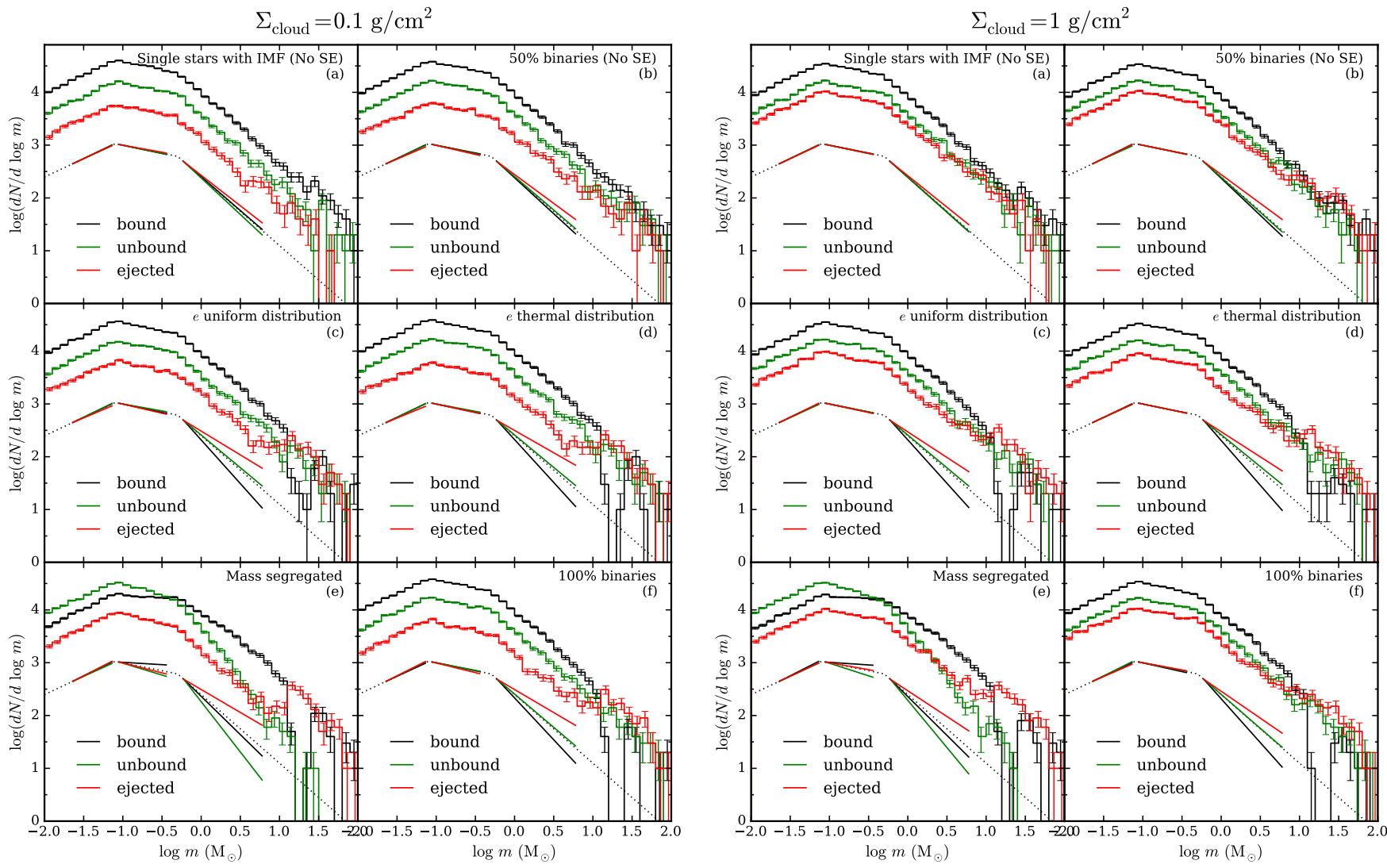

Figure 17. Same as Figure 13, but now for simulations with $\Sigma_{\text {cloud }}=0.1 \mathrm{~g} / \mathrm{cm}^{2}$ (left set of panels) and with $\Sigma_{\text {cloud }}=1 \mathrm{~g} / \mathrm{cm}^{2}($ right set of panels). Labelled panels show the IMFs for the simulation sets: (a) single_imf, (b) binaries_50, (c) binaries_un, (d) binaries_th, (e) segregated and (f) binaries_100. 


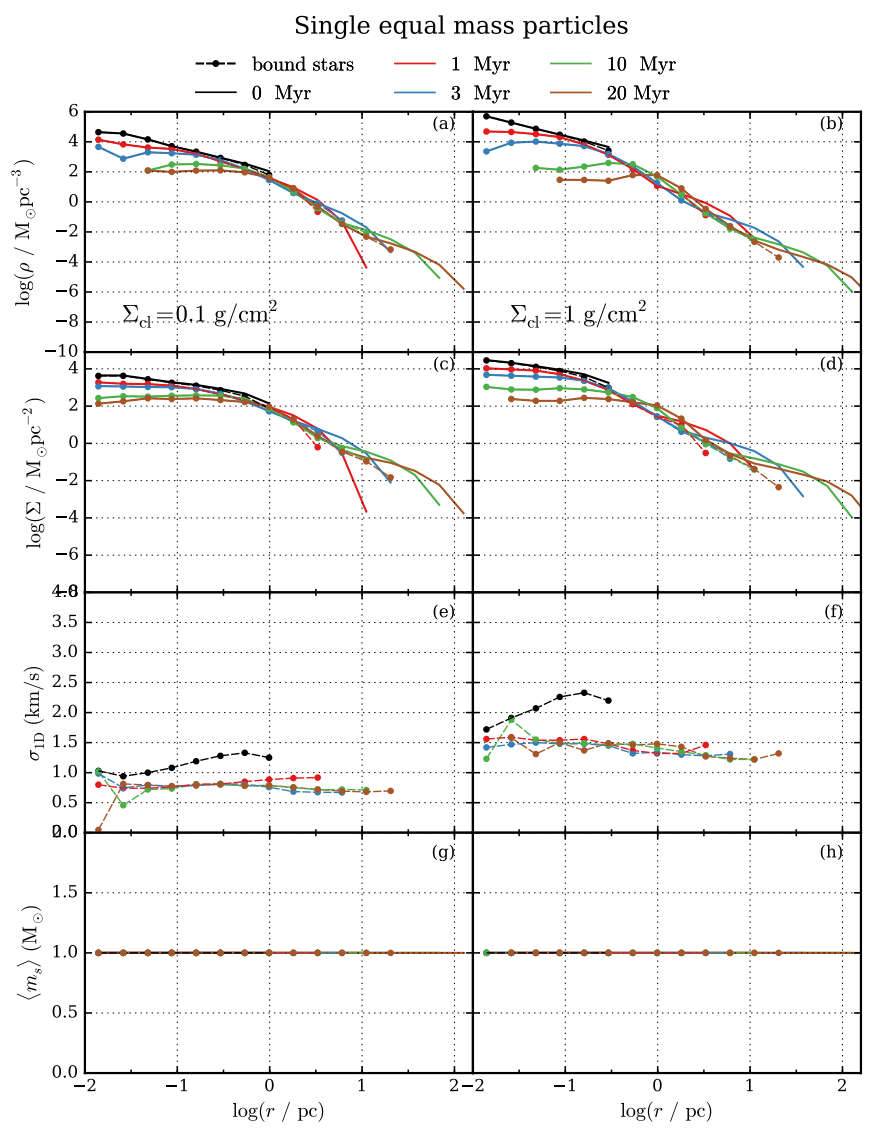

Single stars with IMF (No SE)
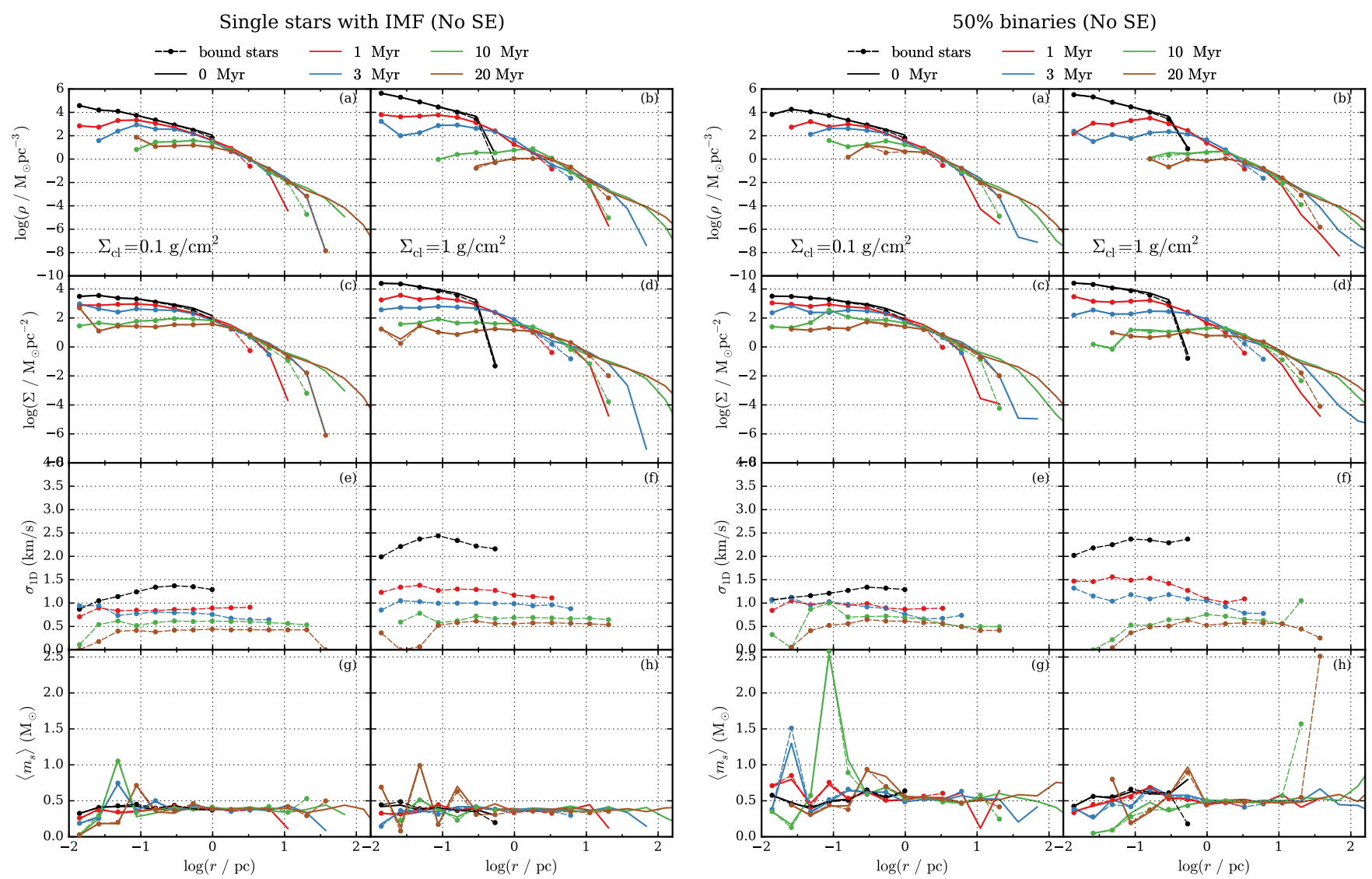

Figure 18. Same as Figure 14, but now showing radial profiles for sets equal_mass (top) single_imf (bottom left) and binaries_50 (bottom right), all with no stellar evolution. 

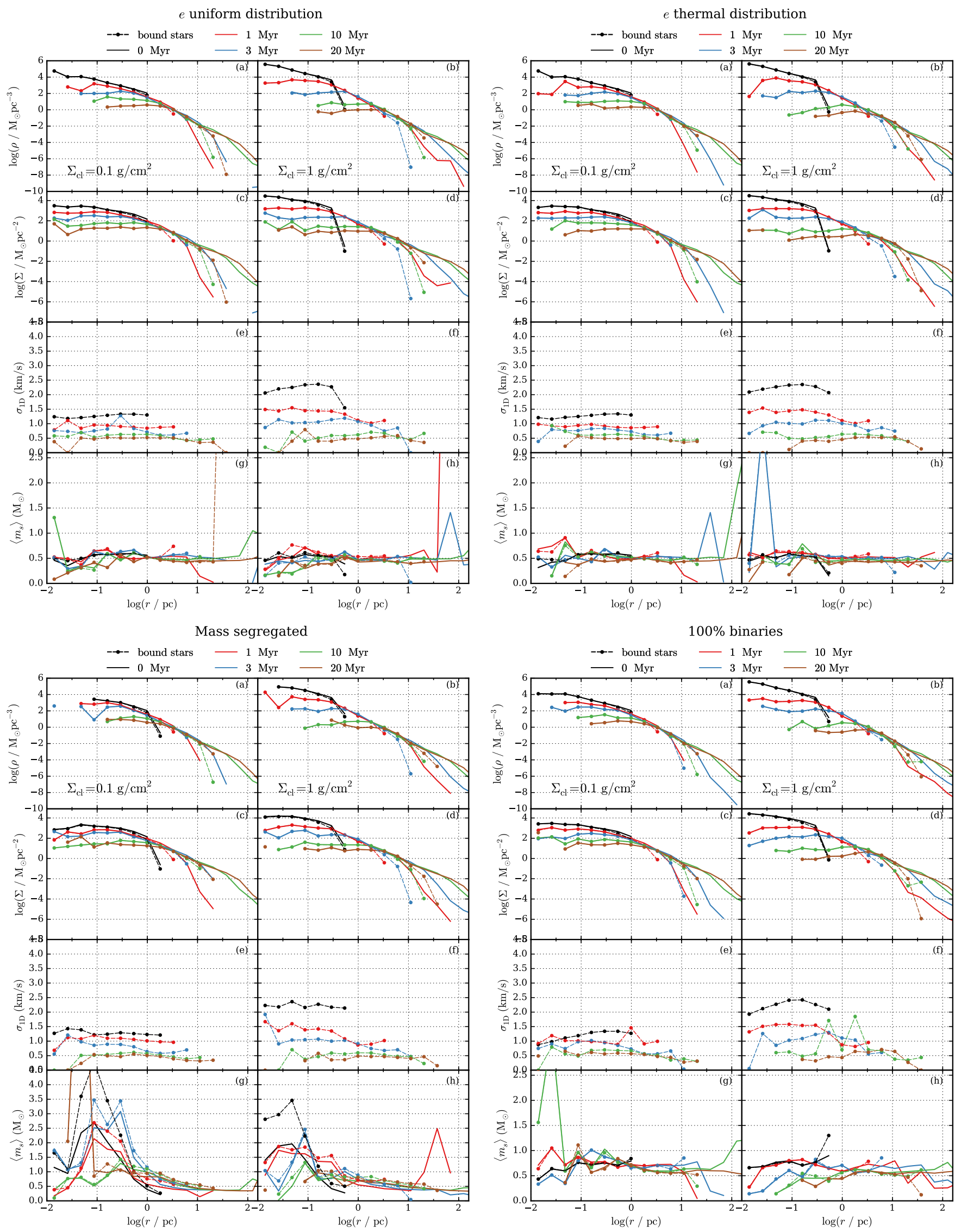

Figure 19. Same as Figure 14, but now for simulations with stellar evolution turned on. Top panels shows simulations with different eccentricity distributions, binaries_un (top left) and binaries_th (top right), and bottom panels shows the most extreme cases: the set with primordial mass segregation segregated (bottom left) and the case with 100 binaries binaries_100 (bottom right). 